\title{
Naegleria's mitotic spindles are built from unique tubulins and highlight core spindle features
}

3 Katrina B Velle ${ }^{1}$, Monika Trupinić ${ }^{2}$, Arian Ivec ${ }^{3}$, Andrew Swafford ${ }^{1}$, Emily Nolton ${ }^{1}$, Luke Rice ${ }^{4}$, Iva 4 M. Tolić ${ }^{2}$, Lillian K Fritz-Laylin ${ }^{1^{*}}$, Patricia Wadsworth ${ }^{1^{*}}$

$5{ }^{1}$ University of Massachusetts, Department of Biology, 611 N. Pleasant St., Amherst MA 01003,

6 USA

7 2 Division of Molecular Biology, Ruđer Bošković Institute, Bijenička cesta 54, 10000 Zagreb,

8 Croatia

$9{ }^{3}$ Department of Physics, Faculty of Science, University of Zagreb, Bijenička cesta 32, 10000

10 Zagreb, Croatia

$11{ }^{4}$ Department of Biophysics, UT Southwestern Medical Center, Dallas, TX 75390, USA

12 * Co-corresponding authors with equal contributions: Ifritzlaylin@umass.edu (L.K.F.-L.),

13 patw@bio.umass.edu (PW)

\section{ABSTRACT}

15 Naegleria gruberi is a unicellular eukaryote whose evolutionary distance from animals and fungi

16 has made it useful for developing hypotheses about the last common eukaryotic ancestor.

17 Naegleria amoebae lack a cytoplasmic microtubule cytoskeleton and assemble microtubules only during mitosis, and thus provides a unique system to study the evolution and functional specificity of mitotic tubulins and the resulting spindle. Previous studies showed that Naegleria amoebae express a divergent $\alpha$-tubulin during mitosis and we now show that Naegleria amoebae express a second mitotic $\alpha$ - and two mitotic $\beta$-tubulins. The mitotic tubulins are evolutionarily divergent relative to typical $\alpha$ - and $\beta$ - tubulins, contain residues that suggest distinct microtubule properties, and may represent drug targets for the "brain-eating amoeba" Naegleria fowleri. Using quantitative light microscopy, we find that Naegleria's mitotic spindle is a distinctive barrel-like structure built from a ring of microtubule bundles. Similar to those of other species, Naegleria's spindle is twisted and its length increases during mitosis suggesting that these aspects of mitosis are ancestral features. Because bundle numbers change during metaphase, we hypothesize that the initial bundles represent kinetochore fibers, and secondary bundles function as bridging fibers. 


\section{INTRODUCTION}

Cells from across the eukaryotic tree use microtubules for a wide variety of functions during both interphase and mitosis. Interphase microtubules play essential roles establishing and maintaining cell shape, polarity, and intracellular trafficking. During cell division, a microtubule-based mitotic spindle self-assembles and mediates chromosome segregation. In most well-studied organisms, the spindle is composed of functionally distinct populations of microtubules, including: (1) kinetochore fiber microtubules that bind to kinetochores to connect each chromosome to a single spindle pole (Inoué and Salmon, 1995); (2) non-kinetochore microtubules that extend from the poles and overlap at the midzone, linking the two halves of the spindle (Mastronarde et al., 1993; Mclntosh, Molodtsov and Ataullakhanov, 2012); and (3) astral microtubules that extend from spindle poles toward the cell cortex. During anaphase, kinetochore microtubules shorten, while midzone microtubules elongate to drive chromosome segregation. A subset of midzone microtubules, called bridging fibers, contact kinetochore fibers in each half spindle (Kajtez et al., 2016). Bridging fibers contribute to the balance of tension and compressive forces in the spindle (Kajtez et al., 2016) and to chromosome motion in anaphase (Vukušić et al., 2017; Vukušić, Buđa and Tolić, 2019). Spindle microtubules are organized by mitotic motor proteins that contribute to microtubule dynamic turnover, spindle pole organization, chromosome congression during prometaphase and poleward motion in anaphase. The influence of motor proteins in spindle structure is highlighted by the twist they introduce in spindles of human cells (Novak et al., 2018).

Interphase and mitotic microtubule functions are emergent properties of microtubule-associated proteins as well as the subunit composition and post-translational modifications of the microtubule polymers themselves. Eukaryotic cells typically express multi-functional tubulins used for both interphase and mitotic functions (Raff, 1984). Human embryonic kidney cells, for example, express high levels of one $\alpha$-tubulin and two $80 \%$ identical $\beta$-tubulins, which are used for both interphase and mitotic functions (Vemu et al., 2017). Similarly, budding yeast express a single $\beta$-tubulin and two $\alpha$-tubulins, which share $88 \%$ sequence identity and are used for both interphase and mitotic functions (Schatz et al., 1986). As an extreme example, the unicellular algae Chlamydomonas has a single $\alpha$ - and a single $\beta$-tubulin gene that are used for all microtubule functions (Johnson, 1998). Other eukaryotes, however, express unique tubulin isotypes that are required for specific microtubule functions, including meiotic spindle assembly in Drosophila oocytes (Matthews, Rees and Kaufman, 1993), axoneme formation in diverse systems (Hoyle and Raff, 1990), and touch receptor neurons in worms (Savage et al., 1989). These specialized tubulins support the "multi-tubulin hypothesis" that posits that different tubulins can specify microtubules with distinct cellular functions (Wilson and Borisy, 1997).

65 The multi-tubulin hypothesis was inspired by studies of Naegleria gruberi-a single-celled eukaryote that diverged from the "yeast to human" lineage over a billion years ago (Fig. 1A)_ with the unusual ability to differentiate from a crawling amoeba to a swimming flagellate (Fig. 1B) (Fulton and Simpson, 1976). The amoeba-to-flagellate differentiation is a stress response that involves the assembly of an entire microtubule cytoskeleton, including centrioles, flagella, and a complete cortical microtubule array. This process includes transcription and translation of flagellate-specific $\alpha$ - and $\beta$ - tubulins along with their associated microtubule binding proteins (Fritz-Laylin, Assaf, et al., 2010). The flagellate state is transient, and cells return to crawling amoebae within 2-300 minutes (Fulton, 1993), after which time the flagellate microtubules are disassembled and tubulin is degraded. This means that the Naegleria flagellate microtubules, and the $\alpha$ - and $\beta$-tubulins that comprise them, are specific for these non-mitotic microtubule 


\section{Naegleria expresses divergent $\alpha$ - and $\beta$-tubulins during mitosis}

104 To determine the number and diversity of tubulins available to Naegleria amoebae and 105 flagellates, we first searched for $\alpha$ - and $\beta$-tubulins in the Naegleria gruberi genome (Fritz-Laylin,

106 Prochnik, et al., 2010). As has been previously reported, we identified 13 a-tubulin and 9

In contrast to most eukaryotic cells, however, Naegleria amoebae have no observable interphase microtubules as visualized by immunofluorescence (Fig. 1B) (Walsh, 2007, 2012), or by electron microscopy (Fulton and Dingle, 1971). Moreover, interphase amoebae lack tubulin transcripts (Lee and Walsh, 1988; Chung et al., 2002). Previous studies have shown that Naegleria has a divergent a-tubulin that is expressed specifically during mitosis (Chung et al., 2002) and that is incorporated into its intra-nuclear mitotic spindle (Walsh, 2007, 2012).

Because Naegleria uses this specific tubulin only for spindle assembly (Chung et al., 2002), it provides a unique opportunity to examine a microtubule system that is specialized for mitosis. The Naegleria spindle also presents an interesting divergent morphology; instead of the typical rod- or fusiform-structures found in many eukaryotes, the Naegleria spindle is barrel-shaped and lacks both conventional kinetochores and obvious microtubule organizing centers (Fulton and Dingle, 1971; Akiyoshi and Gull, 2014; D’Archivio and Wickstead, 2017; Drinnenberg and Akiyoshi, 2017; van Hooff et al., 2017).

Here we test whether-in the absence of the evolutionary constraints imposed by interphase microtubule functions-Naegleria's mitotic microtubule system has diverged from canonical microtubule systems. We show that, in addition to the previously reported mitotic $\alpha$-tubulin, Naegleria expresses a second mitotic $\alpha$-tubulin along with two mitotic $\beta$-tubulins. In contrast to the Naegleria tubulins expressed during the flagellate stage that closely resemble tubulins from heavily-studied species, the protein sequences of the Naegleria mitotic tubulins have diverged significantly and have unique biochemical properties. We use quantitative microscopy to show that mitotic tubulins are used to build an unusual spindle composed of a ring of regularly-spaced microtubule bundles. As mitosis proceeds, additional microtubule bundles form in the equatorial region of the spindle and-as in other eukaryotes-the spindle elongates to facilitate chromosome segregation. The organization and dynamics of the Naegleria spindle highlight both core aspects of mitosis and as well variable features of cell division. $\beta$-tubulin genes, some of which appeared highly divergent, while others are closely related to those of other eukaryotes (Fritz-Laylin, Prochnik, et al., 2010). To further explore the diversity of Naegleria tubulins, we reconstructed a maximum likelihood tree of $\alpha$ - and $\beta$-tubulins using Y-tubulins as an outgroup. Briefly, we collected and aligned 1,191 tubulins sequences from 200 different species (Table S1, Datafile S1), reconstructed a maximum likelihood tree (Fig. S1, Datafile S2), and pruned the resulting tree to more easily visualize the sequences of interest (Fig. 1C, Datafile S3). The tree recovers $\alpha$-tubulins and $\beta$-tubulins as two, monophyletic clades with Naegleria mitotic and flagellar tubulin forming evolutionarily distinct clades within each tubulin family (Fig. 1C).

The Naegleria $\alpha$ - and $\beta$-tubulin sub-clades most closely related to animal and fungal tubulins include those that are expressed during differentiation from the amoeba to the flagellate form (Lai et al., 1979; Lee and Walsh, 1988; Fritz-Laylin and Cande, 2010). These tubulins represent 
the majority of axonemal and cytoplasmic tubulin protein in flagellates (Kowit and Fulton, 1974a, 1974b; Lai, Remillard and Fulton, 1988), and are not expressed in amoebae (Lai et al., 1979; Lee and Walsh, 1988; Fritz-Laylin and Cande, 2010). Flagellate $\alpha$-tubulins are $79-85 \%$ identical to human $\alpha$-tubulin A1B (ENSP00000336799) and flagellate $\beta$-tubulins are $74-75 \%$ identical to human $\beta$-tubulin B1 (ENSP00000217133) (Fig. S2C).

The second Naegleria tubulin sub-clades are more divergent. The second clade of $\alpha$-tubulins contains two sequences from each Naegleria gruberi and Naegleria fowleri, and one from each of the related species Acrasis kona, and Stachyamoeba lipophora. The two N. gruberi a-tubulins are only $57-58 \%$ identical to human $\alpha$-tubulin A1B (Fig. S2C). Similarly, the second clade of Naegleria $\beta$-tubulins also includes $N$. fowleri and $A$. kona sequences, with $N$. gruberi sequences that are $57-58 \%$ identical to human $\beta$-tubulin B1.

Because the ortholog of the previously-reported mitotic a-tubulin (from the NB-1 strain) was among the divergent $\alpha$-tubulins (from strain NEG-M) (Chung et al., 2002; Fritz-Laylin, Prochnik, et al., 2010), we predicted that the divergent Naegleria $\alpha$ - and $\beta$-tubulins are expressed during mitosis. Consistent with this prediction, we compared expression data of amoebae (a population that includes dividing cells) and flagellates and found the conserved tubulins expressed in flagellates and the divergent tubulins expressed in amoebae (Fig. 1D). We confirmed this finding by comparing expression levels of the putative-mitotic tubulins in mitotically synchronized cells to control cell populations and found at least two-fold enrichment of the divergent tubulin transcripts (Fig. S2A-B). Together these data indicate that Naegleria gruberi amoebae expresses divergent $\alpha$ - and $\beta$-tubulins during cell division.

\section{Naegleria mitotic tubulins have diverged in ways that suggest distinct biochemical properties}

Visual inspection of Naegleria mitotic and flagellate tubulin sequences suggested that the mitotic tubulins may have altered microtubule dynamics and/or binding sites for microtubule associated proteins. To more systematically assess this possibility, we quantified the divergence of mitotic and flagellate $\alpha$ - and $\beta$-tubulins as a function of amino acid position. Briefly, after building master multiple sequence alignments for $\alpha$ - and $\beta$-tubulins containing mitotic and flagellate tubulin sequences from $N$. gruberi, $N$. fowleri, and $A$. kona along with reference sequences from more commonly studied organisms (see Methods), we made separate 'mitotic' and 'flagellate' subalignments for each species by only retaining the mitotic or flagellate tubulin from that species (in addition to the reference sequences). We used these subalignments to measure the difference in conservation at each position, and we summarized the results with a positional 'divergence score' (Fig. 2A) in which negative values correspond to greater divergence at a given amino acid position (see Methods). Mitotic $\alpha$-tubulins have more positions with elevated divergence compared to $\beta$-tubulin in all three species (compare Fig. 2A top and bottom), although the absolute number of divergent positions differs by organism (35 positions in a-tubulin vs 23 in $\beta$-tubulin for N. gruberi; 24 vs 22 for N. fowleri; 32 vs 27 for $A$. kona).

Although the positions of elevated variability are distributed throughout the tubulin fold for both $\alpha$ - and $\beta$-tubulin, they appear to be enriched near microtubule polymerization interfaces and surfaces displayed on the inside of the microtubule (Fig. 2B, Fig. S3). To quantify this impression, we tested for enrichment at longitudinal or lateral polymerization interfaces by determining whether the fraction of divergent positions near a given interface was greater than 
162 the fraction of divergent positions across the entire sequence (see Methods). This analysis

163 reveals that divergent positions are more enriched at lateral lattice contacts (2-3-fold increase

164 depending on the species) than at longitudinal lattice contacts (1.1-1.9-fold, depending on the

165 species; Fig. 2C). This enrichment of divergence at lattice interfaces reinforces the idea that

166 microtubules formed from mitotic tubulins will have altered polymerization dynamics and/or

167 distinct structural features.

168 Because fluorescent docetaxel-a microtubule labeling reagent derived from the microtubule-stabilizing drug taxol—appears to bind Naegleria flagellate tubulin but not mitotic tubulin (Fig. 1B), we next examined if taxol-binding residues were conserved in either of these sequences. We focused our analysis on $\beta$-tubulin sequences because that is where taxol binds, and we selected taxol-binding residues based on prior analyses (Gupta et al., 2003) and the structure of taxol-bound microtubules (Alushin et al., 2014). Important taxol-binding amino acids are conserved in flagellate but not in mitotic $\beta$-tubulin sequences (Fig. S4A). Thus, consistent with our observation that fluorescent docetaxel only labels flagellate microtubules, flagellate tubulins appear to have an intact taxol binding site, whereas the mitotic tubulins appear to have lost the ability to bind taxol.

Finally, we noted interesting sequence differences in disordered regions of the Naegleria tubulins. For example, the major site of $\alpha$-tubulin acetylation, $\mathrm{K} 40$, is conserved in the flagellate tubulins, but has diverged in the mitotic tubulins (Fig. S4B). We also characterized the length and predicted net charges of the C-terminal tubulin tails (Fig. S4C). The tubulin tails of both mitotic and flagellate $\alpha$-tubulins have lengths and net changes similar to those observed in more commonly studied tubulins. In contrast, the mitotic $\beta$-tubulin tails are slightly less charged than their flagellate counterparts (Fig. S4C). Finally, the C-terminal EY sequence in $\alpha$-tubulin that is recognized by regulatory factors that contain a CAP-GLY domain is notably absent from both flagellate and mitotic tubulin sequences, suggesting differences in their regulation (Fig. S4C). Together, these observations reinforce the notion that microtubules assembled from mitotic $\alpha \beta$-tubulins are likely to have different polymerization dynamics and/or binding partners compared to microtubules assembled from flagellate $\alpha \beta$-tubulins.

\section{The Naegleria spindle is a hollow barrel of microtubule bundles that elongates as mitosis proceeds}

To explore whether the sequence divergence of Naegleria's mitotic tubulins translates into a divergent organization of mitotic microtubules, we fixed Naegleria amoebae undergoing closed mitosis. We stained mitotic microtubules with anti-tubulin antibodies and DNA with DAPI, and visualized the cells using spinning disk confocal microscopy (Fig. 3). Consistent with previous results (Fulton and Dingle, 1971; Chung et al., 2002; Fritz-Laylin et al., 2011; Walsh, 2012), we find that the Naegleria spindle is composed of microtubule bundles and lacks obvious microtubule organizing centers (Fig. 3). The microtubule bundles appear to form around a ball of DNA; we refer to this stage as prophase (Fig. 3A). This cage-like array of microtubule bundles reorganizes into a barrel-shaped spindle with DNA aligned in a broad, hollow band at the midplane; we refer to this stage as metaphase (Fig. 3A). Although in some cases the spindle has a tapered morphology (Fig. 3A, left metaphase cell) the majority of spindles are characterized by broad, flat poles (Fig. 3A, middle and right metaphase cells). We also observed spindles in which the DNA is segregated to the ends of the elongated spindle, which we classified as anaphase/telophase. Compared with other stages of mitosis, few spindles were 
detected during the early stages of chromosome segregation, suggesting that this stage occupies a small fraction of the total duration of mitosis. In contrast, cells with elongated spindles and segregated DNA were relatively common, suggesting that the late anaphase spindle is stable for some time. By quantifying spindle length and width, we infer that spindle length increases while width decreases as mitosis progresses from prophase to anaphase/telophase (Fig. 3B).

Because mitotic cells were relatively rare in asynchronous populations, we also examined mitotically synchronized cells (Fulton and Guerrini, 1969) (Fig. S5), and found no qualitative or quantitative differences in spindle microtubule organization between the synchronized and asynchronous cells (Fig. S5C). This supports previous reports that synchronization does not alter spindle morphology in Naegleria amoebae (Fulton and Guerrini, 1969). We therefore used cells from both synchronized and asynchronized populations for the following analyses.

To determine the organization of microtubule bundles in the Naegleria spindle, we visualized axial and transverse slices of spindles oriented both parallel and perpendicular to the coverslip (Fig. 3C-D). These analyses confirmed that the microtubules in the Naegleria metaphase spindle are organized in a ring, similar to the staves of a barrel (Fig. 3C-D). Previous studies have suggested that this barrel is assembled around the nucleolus, which remains intact during mitosis (Naegleria's ribosomal RNA genes are encoded on a plasmid that does not condense during prophase (Fritz-Laylin et al., 2011; Walsh, 2012)). To confirm the retention of the nucleolus during mitosis, we co-stained cells with anti-nucleolar and/or anti-tubulin antibodies, as well asDAPI to visualize DNA (Fig. 3E). Consistent with previous work, we find that the nucleolus remains throughout mitosis, at times encompassing much of the spindle volume (Walsh, 2012). The nucleolus divides before chromosome segregation, resulting in one nucleolus at each end of the spindle with the chromosomes nestled between them in a thin disk (Fig. 3E).

Comparing the dimensions and intensity of the microtubule arrays in flagellates to those in mitotic cells suggests that the spindle is composed of bundles rather than individual microtubules (Fig. 1B). Supporting this idea, we observed a single anaphase cell in which a microtubule bundle appears to have splayed apart, revealing at least five fluorescent elements which may represent individual microtubules (Fig. S5E). To estimate the number of microtubules per bundle, we fixed Naegleria amoeba for thin section transmission electron microscopy. Longitudinal sections through mitotic cells reveal that bundles are composed of multiple, closely-associated individual microtubules (Fig. 3F). We observed three to six microtubules in a single longitudinal section consistent with previous estimates in the related $N$. fowleri (González-Robles et al., 2009). In summary, our data show that the Naegleria spindle is composed of a ring of microtubule bundles that elongates during chromosome segregation.

\section{Naegleria spindles have two sets of microtubule bundles}

Although most spindles were oriented parallel to the coverslip surface, some spindles were oriented perpendicular to the coverslip, providing improved resolution of the microtubule bundles (Fig. 4A). These end-on views revealed variation in the number of microtubule bundles (Fig. 4A-C). Some spindles have a single ring of approximately 12 evenly-spaced bundles with $0.79 \mu \mathrm{m}$ center-to-center spacing (range: 0.42-1.90; SD: 0.28; $\mathrm{n}$ : 31 measurements from 3 spindles). These "primary bundles" extend the entire length of the spindle (Fig. 4A, left, Fig. 4B, 
249 top). Other spindles, however, have additional bundles adjacent to the main ring (Fig. 4A, 250 middle and right, Fig. 4B bottom). Importantly, the number of bundles in this second class of 251 spindles varied along the spindle axis, with additional "secondary bundles" restricted to the 252 spindle midplane with the primary bundles extending out to the spindle ends.

253 If the secondary bundles were formed from new microtubule polymerization, we would expect 254 the mid-region of metaphase spindles to have a greater amount of tubulin than the poles. We 255 therefore quantified tubulin and DNA fluorescence intensity along horizontally-oriented spindles at each stage of mitosis (Fig. 4D, Fig. S6). The total amount of tubulin within the spindle increases as mitosis proceeds, consistent with microtubule assembly (Fig 4E). Metaphase spindles show variable tubulin distributions (Fig. 4D), with a subset having a clear peak of intensity toward the spindle midzone with "shoulders" on either side (Fig. 4D, rightmost metaphase). This pattern is reminiscent of the larger number of bundles that we quantified at the centers of vertically oriented spindles (Fig. 4B), and is consistent with secondary bundle formation involving additional microtubule assembly. Although this subset of metaphase spindles had clear "shoulders" in their tubulin distributions, other distributions were less clear-cut (Fig. 4D, center metaphase panel). The variability in the tubulin distribution across metaphase spindles raises the possibility that secondary bundles may form asynchronously within a spindle, consistent with cross sections of vertically-oriented spindles that show only a few secondary bundles (Fig. 4A, middle cell). By quantifying the maximum number of bundles per vertically-oriented spindle (Fig. $4 \mathrm{C}$ ), we found that the maximum bundle number varies from $\sim 10$ to 25 , with many cells showing intermediate values. This continuous distribution is consistent with asynchronous secondary bundle assembly rather than the two distinct populations we would expect for a synchronous event.

272 Finally, we examined the tubulin distribution in anaphase and telophase cells to determine the 273 fate of the secondary bundles that form during metaphase. Although the tubulin intensity in 274 these spindles was relatively uniform across the spindle midzone, we observed distinct peaks at each end of the spindle, indicating a higher density of microtubules (Fig. 4D, anaphase), consistent with both primary and secondary bundles remaining associated with chromosomes throughout mitosis. Together, these data suggest that secondary microtubule bundles assemble asynchronously during metaphase by new microtubule assembly and may persist through late mitosis. Based on these and other data, we hypothesize that primary bundles serve as kinetochore fibers and secondary bundles as bridging fibers (see Discussion).

\section{The Naegleria spindle twists from pole-to-pole in a right-handed fashion}

282 The 3D reconstructions of vertically-oriented spindles revealed that the microtubule bundles 283 curved and appeared to twist from one end of the spindle to the other (Fig. 4A, Fig. 5A, Movie 284 S1, Movie S2). To quantify this, we traced individual bundles of metaphase spindles (Fig. 5A) 285 and measured their curvature and twist by fitting a plane to the points representing the bundle 286 and a circle that lies in this plane to the same points. We then estimated bundle curvature as 287 one over the radius of the fit circle, and the twist as the angle between the plane and the $z$-axis 288 divided by the mean distance of these points from the z-axis (Fig. 5B).

289 The resulting data show that microtubule bundles in the Naegleria spindle are curved $(0.146 \pm$

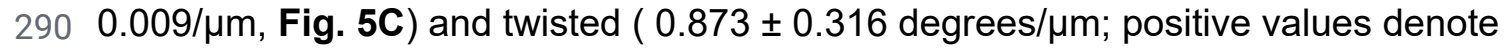


right-handed and negative values left-handed twist Fig. 5D), with shorter bundles having more curve and twist than longer bundles (Fig. 5C-D). On average, the bundles were twisted in a right-handed direction, making the spindle a chiral structure with right-handed asymmetry. This result was corroborated by visual assessment of the handedness of the spindle twist. Here, if the bundles rotate counterclockwise when moving along the spindle axis in the direction towards the observer, the twist is right-handed, and vice versa. We found a mixture of left- and right-handed twist, with the majority of spindles showing a strong right-handed twist (Fig. 5E). Analyzing early metaphase (defined as cells with $<20$ bundles) and late metaphase (defined as cells with $>20$ bundles) cells separately suggests that bundles increase in length and decrease in curvature during metaphase (Fig. S7A and S7D). Right-handed twist was dominant for vertically- and horizontally-oriented spindles, and for cells in early and late metaphase (Fig. S7B and G), suggesting that the handedness of spindle chirality does not depend on mitotic stage or spindle orientation during imaging. Together, these data indicate that the microtubule bundles are physically linked and under rotational forces.

Other than HeLa cells, Naegleria are the only cell type whose spindle twist has been measured. The microtubule bundles of Naegleria's spindle are less curved than those of human HeLa cell spindles, as the radius of curvature is larger for Naegleria, $6.9 \pm 0.4 \mu \mathrm{m}$, than for the outermost bundles in HeLa cells, $5.1 \pm 0.3 \mu \mathrm{m}$ (Manenica et al., 2020). Moreover, the radius of curvature normalized to the spindle half-length, which is equal to 1 for bundles shaped as a semicircle, is $1.26 \pm 0.05$ for Naegleria and $0.90 \pm 0.05$ for HeLa cells (Manenica et al., 2020), also indicating a smaller curvature of Naegleria spindles. In line with the smaller curvature, the absolute value of the average spindle twist in Naegleria is smaller than in HeLa cells, $0.9 \pm 0.3$ degrees/ $\mu \mathrm{m}$ in Naegleria vs. 2 degrees/um in HeLa (Novak et al., 2018). Yet, twist of Naegleria spindles is more eye-catching than in HeLa cells, due to the smaller number of microtubule bundles, which are well-defined and have a uniform shape, in contrast to the less ordered distribution and shapes of bundles in HeLa cells.

\section{DISCUSSION}

Naegleria amoebae represent a remarkable system with which to study microtubule biology because they do not have interphase microtubules. Naegleria is not the only species without interphase microtubules; the cytoplasm of interphase Entamoeba histolytica amoebae also has no observable microtubules (Meza, Talamás-Rohana and Vargas, 2006). In contrast to Entamoeba, however, Naegleria can differentiate into a secondary cell type, the flagellate. Here we show that Naegleria express unique tubulins in mitotic amoebae that are distinct from the tubulins expressed in flagellate cells. While flagellate tubulins—used to assemble both flagellar and cytoplasmic microtubules (Fulton and Kowit, 1975; Fulton and Simpson, 1976; Fulton, 1983; Lai, Remillard and Fulton, 1988; Fritz-Laylin and Cande, 2010)_are highly similar to tubulins of other eukaryotic species, the mitotic tubulins have diverged in sequence, including at key residues likely to alter microtubule structure or dynamics. Because the sequence similarity between Naegleria and Acrasis flagellate tubulin isoforms is much higher than their mitotic tubulins (Fig. S2-C), we infer that the cytoplasmic functions of tubulins may require more stringent sequence conservation than mitotic functions.

Naegleria mitotic microtubules assemble into a hollow, barrel-shaped mitotic spindle comprising distinct bundles, each made of multiple microtubules. Based on these observations and additional literature discussed below, we propose the following model for Naegleria spindle 
elongation and chromosome segregation (Fig. 6): (1) Mitosis begins with the assembly of "primary" microtubule bundles. Each primary bundle is associated with a chromosome and functions as a pair of kinetochore fibers; (2) During metaphase, "secondary" microtubule bundles form near the spindle midplane that function as bridging fibers, connecting kinetochore fibers associated with sister chromatids (Simunić and Tolić, 2016; Vukušić et al., 2017); (3) chromosome-to-pole motion occurs as primary bundles depolymerize while secondary bundles elongate to form the spindle midzone and further separate the chromosomes. Under this model, the higher microtubule density toward the poles during late anaphase results from fluorescence of both kinetochore and bridging fiber microtubules. While this model is consistent with our quantitative measurements, other scenarios are also possible. For example, secondary bundles could associate with chromosomes, functioning like kinetochore fibers, and primary bundles could form the midzone, or each individual bundle could be composed of bridging and kinetochore fiber microtubules, that ultimately sort into the anaphase spindle.

The possibility that primary bundles function as kinetochore fibers is consistent with our previous estimate of $\sim 12$ chromosomes in Naegleria (Fritz-Laylin, Prochnik, et al., 2010), a value that is similar to the average number of primary bundles we observe in early metaphase spindles (Fig. 4). We also observe "kinks" in the center of some spindles suggesting that each primary bundle may be composed of two kinetochore fibers. Although conventional, trilaminar kinetochores have not been detected using electron microscopy (Fig. 3F) (Fulton and Dingle, 1971), homologs of a subset of kinetochore proteins identified in other organisms are present in the Naegleria genome (Akiyoshi and Gull, 2014; van Hooff et al., 2017), hinting at the presence of yet-to-be-detected kinetochores. Whether or not Naegleria has conventional kinetochores, spindle assembly and chromosome movement is well established to occur in the absence of kinetochores (Heald et al., 1996; Brunet et al., 1999). For example, in both mouse and C. elegans meiotic and human mitotic spindles, lateral interactions between microtubules and chromosomes drive chromosome congression, although chromosome-to-pole motion does require kinetochore-microtubule interactions (Kapoor et al., 2006; Mullen, Davis-Roca and Wignall, 2019; Danlasky et al., 2020).

Our working model posits that both anaphase A, chromosome-to-pole motion, and anaphase B spindle elongation, contribute to chromosome segregation in Naegleria. The presence of short microtubule bundles between the chromosomes and poles in anaphase spindles is consistent with microtubule depolymerization during anaphase $A$, although the location and regulation of microtubule assembly and disassembly in these cells is not yet known. Anaphase/telophase spindles in Naegleria are longer than metaphase spindles, consistent with anaphase B spindle elongation. In meiotic spindles, anaphase B is driven, at least in part, as polymerizing midzone microtubules interact with chromosomes (Dumont, Oegema and Desai, 2010; Danlasky et al., 2020). In mammalian cells, links between elongating midzone bridging microtubules and kinetochore fibers contribute to anaphase (Vukušić et al., 2017; Vukušić, Buđa and Tolić, 2019). Although the mechanism of spindle elongation in Naegleria is not yet established, the appearance of secondary bundles in the chromosome region is reminiscent of bridging fibers in other cell types (Simunić and Tolić, 2016). This similarity suggests that interactions between primary and secondary microtubule bundles may contribute to chromosome segregation (Vukušić, Buđa and Tolić, 2019).

These bundles differentiate the Naegleria spindle from those of other species that typically contain both individual and bundled microtubules. Despite this difference, microtubule bundles 
in Naegleria and human cells both show twist, suggesting that this may be a conserved feature of eukaryotic spindles. In contrast to the left-handed chirality previously measured in human spindles (Novak et al., 2018), the majority of Naegleria spindles are right-handed. Because Naegleria is only the second species whose spindle chirality has been measured, it is difficult to know whether its chirality is unusual. Regardless, the requirement of the motor activity of kinesin Eg5 in the twisting of human spindles suggests that Naegleria spindle twist may also depend on the activity of microtubule motors that generate torque within the bundles (Tolić, Novak and Pavin, 2019).

Naegleria's evolutionary position makes it uniquely suited for identifying features of mitotic spindles that may be deeply conserved, including their bi-polarity, elongation, and twist. Naegleria's position also highlights features that may be lineage-specific due to their absence in this distant species. For example, some features of animal cell spindles are missing from Naegleria, including obvious microtubule organizing centers as well as astral microtubules which contribute to spindle position and to cytokinesis in other cells. Whether these differences are related to the divergence of the Naegleria mitotic tubulins awaits further investigation.

The unique properties of these mitotic tubulins may also have practical value. Although the model species Naegleria gruberi is innocuous, the related Naegleria fowleri is the infamous "brain-eating amoeba" that causes a devastating and usually lethal brain infection (Siddiqui et al., 2016). Because the divergent residues we have identified in the Naegleria mitotic tubulins are conserved in both Naegleria species but not in human tubulins (Fig. 2, Fig. S4), these residues represent potential targets for specific therapeutics that could disrupt Naegleria cell division to halt in vivo growth.

\section{MATERIALS AND METHODS}

Phylogenetic tree estimation: To establish a more inclusive comparison of Naegleria $\alpha-$, and $\beta$-tubulins to those of other eukaryotes, 1,191 tubulins from 200 different species were analyzed (Table S1), adding sequences from Naegleria gruberi (Fritz-Laylin, Prochnik, et al., 2010), Naegleria fowleri (Herman et al., 2020), and Acrasis kona (personal communication, Sandra Baldauf, Uppsala University) to those identified as $\alpha, \beta$, and $y$ tubulins using the PhyloToL pipeline (Cerón-Romero et al., 2019). Prior to alignment, sequences from the same species that were $100 \%$ identical were removed, leaving only one copy before re-merging the datasets. Sequences were aligned using the PASTA iterative alignment algorithm with the MUSCLE algorithm as the aligner and merger (Mirarab et al., 2015). IQ-Tree v1.16.2 was used for model selection, which indicated LG4M+R10 as the best model for reconstruction (Kalyaanamoorthy et al., 2017; Minh et al., 2020). Due to the size of the tree, LG4M was used balance the accuracy of tree solving and the constraints of modern processing power. A maximum likelihood tree was reconstructed using IQ-Tree with 10,000 ultrafast bootstraps (Hoang et al., 2018). 1,000 bootstraps of the approximate likelihood ratio test (Guindon et al., 2010) as well as the aBayes test (Anisimova et al., 2011) were then used to further test node support. The ITOL web server was used for tree visualization (Letunic and Bork, 2019).

Characterization of Naegleria mitotic tubulin sequences: To quantify the divergence of mitotic and flagellate $\alpha$ - and $\beta$-tubulins from $N$. gruberi, $N$. fowleri, and $A$. kona as a function of 
amino acid position, we compared them to a common reference consisting of sequences of $\alpha$ or $\beta$-tubulin sequences from commonly studied model organisms (Homo sapiens, Sus scrofa, Bos taurus, Drosophila melanogaster, Mus musculus, Saccharomyces cerevisiae, Schizosaccharomyces pombe, and Chlamydomonas reinhardtii). Multiple sequence alignments were first prepared for $\alpha$ - and $\beta$-tubulin using ClustalOmega (Madeira et al., 2019). These 'master' alignments contained the reference sequences as well as mitotic and flagellate sequences from the three species of interest. Separate "flagellate" and "mitotic" subalignments were then prepared for each species by only retaining flagellate or mitotic sequences from a given species, in addition to the common reference sequences. We quantified sequence conservation/divergence as a function of amino acid position in these subalignments using the AL2CO server (Pei and Grishin, 2001), using normalized sum of pairs scoring (BLOSUM62 weighting) and otherwise default settings. The resulting conservation scores are normalized so that completely conserved positions return the same score regardless of the identity of the conserved amino acid; lower scores (including negative scores) correspond to less conservation. To assess differences in conservation between mitotic and flagellate sequences, the flagellate score was subtracted from the mitotic score at each amino acid position. The resulting difference score is close to zero when a position in the mitotic and flagellate sequences is equally conserved/diverged relative to the set of references sequences; it is positive when the mitotic sequence is less divergent, and negative when the mitotic sequence is more divergent. To identify the positions where the divergence of mitotic sequences was greater than flagellate sequences, the conservation score at each position was divided by the standard deviation of scores over all positions. We focused our subsequent analysis on especially divergent positions, which we defined as those where the relative divergence was greater than two standard deviations away from the mean (Fig. 2A).

We used PyMol (citation: The PyMOL Molecular Graphics System, Version 2.4.1 Schrödinger, LLC) and a cryo-EM structure of $\alpha \beta$-tubulin in a microtubule (PDB code 6O2R (Eshun-Wilson et al., 2019)) to assess if the especially divergent positions in mitotic tubulins were enriched near microtubule polymerization interfaces (Fig. 2B-C, Fig. S3). To obtain the overall fraction of especially divergent positions per chain, the number of especially divergent positions in $\alpha$ - and $\beta$-tubulin was divided by the total number of amino acids. To calculate the proportion of divergent positions near lateral or longitudinal interfaces, we used distance based selections to identify the amino acids within a cutoff distance of a lateral or longitudinal lattice neighbor, and calculated the ratio of divergent to total positions within this subset.

Cell and bacterial culture: Naegleria amoebae (strain NEG, ATCC strain 30223) and their food source Aerobacter aerogenes (a gift from the laboratory of Chandler Fulton, Brandeis University) were routinely cultured following previously established protocols (Heuser and Razavi, 1970). Briefly, $A$. aerogenes were regularly streaked from a frozen glycerol stock, and single colonies were grown stationary at room temperature in penassay broth (Difco antibiotic medium 3). Liquid cultures were used to grow lawns of $A$. aerogenes overnight on NM plates (2 g/L Difco Bacto peptone, 2 g/L glucose, 1.5 g/L K2HPO4, 1 g/L KH2PO4, 20 g/L agar). Lawns were inoculated with a loopful of NEG amoebae or cysts to create an edge plate (from a previous edge or cyst plate). Plates were sealed with parafilm, inverted, and incubated for 1-3 days at $28{ }^{\circ} \mathrm{C}$. For starvation-induced differentiation (Fig 1B), cells were shocked with ice cold 2 $\mathrm{mM}$ Tris, and transferred to a shaking flask at $28{ }^{\circ} \mathrm{C}$ for $1 \mathrm{~h}$.

Mitotic synchronies: To obtain a population of synchronized cells, we modified a previously 
466 published method (Fulton and Guerrini, 1969) to cause a heat-induced mitotic arrest. Briefly, the 467 day before the synchrony, a lawn of $A$. aerogenes was collected in $10 \mathrm{ml}$ of TrisMg ( $2 \mathrm{mM}$ Tris + $46810 \mathrm{mM} \mathrm{MgSO} 4$ ), pelleted, resuspended in $20 \mathrm{ml}$ TrisMg. $10 \mathrm{ml}$ of the bacterial solution were 469 transferred into a $125 \mathrm{ml}$ flask. $2-8 \times 10^{\wedge} 5$ amoebae were added to the flask and covered with 470 foil, and the culture was incubated in a shaking water bath overnight ( $\left.125 \mathrm{RPM}, 30^{\circ} \mathrm{C}\right)$. The 471 morning of the synchrony, two additional lawns of $A$. aerogenes were collected, pelleted, and 472 resuspended in $40 \mathrm{ml}$ TrisMg. This solution was added to the flask with Naegleria, and allowed 473 to shake for 3 minutes to thoroughly mix. This mixture was divided into 2 new (uncovered) 474 flasks, one "control" and one "experimental," and cell counts were taken with a hemocytometer. 475 Cells were counted approximately every $20 \mathrm{~min}$, and once the cells had doubled from their 476 starting concentration, a sample was taken for quantitative real time PCR (qPCR) analysis (see 477 next section), and the experimental flask was moved to a $38.5+/-0.5{ }^{\circ} \mathrm{C}$ water bath. Cells were 478 counted from each flask, and when the control flask had doubled again, another sample was 479 taken from each flask for qPCR, and then the experimental flask was shifted back to $30^{\circ} \mathrm{C}$. 480 Samples were taken from the experimental flask after shifting back to $30^{\circ} \mathrm{C}$ to fix and stain cells 481 for mitotic spindles.

482 Analysis of tubulin gene expression: Samples were collected from each flask prior to the 483 temperature shift (pre-shift, control and experimental flasks), and again after incubation at $38^{\circ} \mathrm{C}$ 484 (or $30{ }^{\circ} \mathrm{C}$ for the control flask) but before shifting back to $30^{\circ} \mathrm{C}$. For each sample, $5 \mathrm{ml}$ of cells 485 were spun down at $1500 \mathrm{RCF}$ at $4{ }^{\circ} \mathrm{C}$ for $5 \mathrm{~min}$ and the supernatant was discarded. The cell 486 pellet was suspended in $1 \mathrm{ml} \mathrm{TRIzol}$, vortexed, and promptly stored at $-80^{\circ} \mathrm{C}$ until RNA 487 extractions. Cells were lysed using FastPrep homogenizer with bead beating in TRIzol. Lysate 488 was cleaned up using a Zymo kit with on column DNase treatment, and RNA was eluted in $30 \mu \mathrm{l}$ 489 of kit-provided water. cDNA libraries were then generated using a Thermo Fisher/Invitrogen

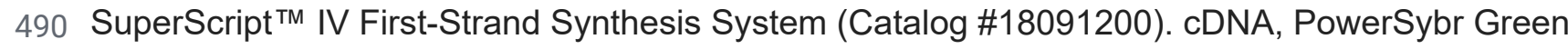
491 (Thermo Fisher: 4368706), and primers were mixed in triplicate in a MicroAmp ${ }^{\text {TM }}$ Fast Optical 492 96-Well Reaction Plate with Barcode (Catalog \#4346906) and sealed with an optical adhesive 493 cover (Catalog \#4360954). Primer sequences were as follows: GAPDH (JGI ID: 53883): forward 494 TGGCTCCAATTGCTGCTGTTT, reverse CCTTAGCAGCACCAGTTGAAGA; G protein (77952): 495 forward ACGGTTGGGTCACTTGTTTGTCC, reverse GAGCGTGACCAGTGAGGGATC; mitotic 496 a-tubulin (58607): forward GGTCCTTGATGTGTGCCGAAC, reverse

497 TTAGCAGCATCTTCACGACCAGT; mitotic $\alpha$ - tubulin (55745) forward

498 CACACACAAAATGAGAGAAGTCGTC, reverse TTCCATGTTCAGCACAGAATAATTC; mitotic 499 B-tubulin (55748): forward AACCAACACTGCTTCTCCACTCG, reverse

500 TCTGGACGGAATAATTGACCTTGG; mitotic $\beta$-tubulin (55900): forward

501 GGTTGCTGGTGTCATGTCTGGTG, reverse GCAGCCAAAGGAGCAGAACCAA. Samples

502 were run on a StepOne Real-Time PCR machine and analyzed using StepOne software v2.3.

503 The fold change in mRNA abundance was determined from $C_{T}$ values using the $2^{-\Delta \Delta C t}$ method 504 (Livak and Schmittgen, 2001). Using this method, the flask that remained at $30^{\circ} \mathrm{C}$ was a 505 time-matched control for the experimental flask at the time point before the temperature shift, 506 and the time point after the shift to $38^{\circ} \mathrm{C}$. A Naegleria $\mathrm{G}$ protein was used as the housekeeping 507 gene to normalize the data, and a second housekeeping gene (GAPDH) was used to verify the 508 results.

509 The microarray data in Fig. 1D was originally acquired in (Fritz-Laylin and Cande, 2010). Each 510 experimental replicate had been completed with 2 technical replicates, so the technical 
replicates were first averaged. Then, the mRNA abundance at the 0 min time point (before differentiation) and at the 80 min time point (after differentiation to flagellates) were compared for each biological replicate to calculate the fold change in mRNA abundance for mitotic and flagellate tubulins.

Immunofluorescence: Immunofluorescence staining of amoebae and flagellates in Fig 1B was performed using an actin cytoskeleton fixation protocol modified from (Velle and Fritz-Laylin, 2020). Cells were taken from an edge plate or from a sample of differentiated cells (see above), spun down at $1500 \mathrm{RCF}$ for $90 \mathrm{sec}$, and cell pellets were resuspended in $1.5 \mathrm{ml} 2 \mathrm{mM}$ Tris. Cells were fixed in an equal volume of $2 x$ fixative $(50 \mathrm{mM}$ sodium phosphate buffer, $125 \mathrm{mM}$ sucrose, and $3.6 \%$ paraformaldehyde) for 15 minutes, then transferred to a 96 well glass-bottom plate coated with $0.1 \%$ poly(ethyleneimine) and allowed to settle for $15 \mathrm{~min}$. Cells were rinsed twice in PEM (100 mM PIPES, 1 mM EGTA, $0.1 \mathrm{mM} \mathrm{MgSO4;} \mathrm{pH} ~ 7.4$ ) and permeabilized for 10 min in PEM + 0.1\% NP-40 Alternative (Millipore, 492016) + 6.6 nM Alexa Fluor ${ }^{\text {TM }} 488$ Phalloidin (and 0.2x Tubulin Tracker Deep Red (Life Technologies, T34077, prepared according to manufacturer instructions) columns 1, 2 and 4 only). Cells were rinsed twice in PEM, then blocked in PEMBALG (PEM + 1\% BSA, $0.1 \%$ sodium azide, $100 \mathrm{mM}$ lysine, and $0.5 \%$ cold fish water gelatin; $\mathrm{pH} 7.4$ ) at room temperature for $1 \mathrm{~h}$. Cells were then incubated in primary antibody (anti-a-tubulin mouse monoclonal antibody (clone DM1A), Sigma, T6199) diluted to $\sim 10 \mu \mathrm{g} / \mathrm{ml}$ in PEMBALG for $1 \mathrm{~h}$. Cells were washed 3 times in PEMBALG, then incubated at room temperature for $1 \mathrm{~h}$ in Alexa Fluor ${ }^{\mathrm{TM}} 555$ conjugated goat anti-mouse secondary antibody (Life Technologies, A21424) diluted to $2 \mu \mathrm{g} / \mathrm{ml}$ in PEMBALG, with 1x Tubulin Tracker Deep Red, $\sim 66 \mathrm{nM}$ Alexa Fluor ${ }^{\mathrm{TM}} 488$ Phalloidin, and $1 \mu \mathrm{g} / \mathrm{ml}$ DAPI. Cells were then rinsed 4 times in PEM, and imaged the same day.

Immunofluorescence staining in the remaining figures was optimized for microtubules and performed using amoeba from a fresh edge plate that had grown about half-way across the dish (or from a mitotic synchrony, detailed above). Cells were removed from the plate and added to approximately $3 \mathrm{mls}$ of water in a conical tube, spun down in a clinical centrifuge at setting 7 for $\sim 40$ seconds and the supernatant removed leaving $\sim 500 \mu \mathrm{l}$ of water above the cell pellet. To this mixture an equal volume of freshly prepared $2 \mathrm{X}$ fixative solution consisting of $2 \mathrm{mM}$ Tris $\mathrm{pH} 7.2$; $125 \mathrm{mM}$ sucrose; $10 \mathrm{mM} \mathrm{NaCl}, 2 \%$ paraformaldehyde was added and mixed gently. Cells were fixed for $10 \mathrm{~min}$ at room temperature. Cells were then placed on freshly coated coverslips and allowed to adhere for approximately 20-30 minutes. Coverslips were plasma cleaned and then coated with $0.1 \%$ poly(ethyleneimine). After cells were adhered to the coverslips, they were rinsed 3 times with $1 \mathrm{ml}$ of PEM (100 mM PIPES, pH 6.9; 1 mM EGTA; $0.1 \mathrm{mM} \mathrm{MgSO} 4$ ) and then permeabilized with $0.1 \%$ NP-40 for 10 minutes. Cells were blocked in PEM-BALG (PEM buffer supplemented with $1 \%$ BSA, $100 \mathrm{mM}$ lysine, and $0.5 \%$ cold fish water gelatin) for one hour or overnight and then incubated with primary antibody for 1 hour at $37^{\circ} \mathrm{C}$ or at room temperature overnight. Coverslips were rinsed in PBS containing $0.1 \%$ Tween and $0.02 \%$ sodium azide and incubated with Dylight-488 labeled anti-mouse secondary antibodies (Invitrogen) according to the manufacturers' recommended protocol. Finally, coverslips were washed in PEM supplemented with $0.01 \%$ Triton-X-100 for 5 minutes before mounting on clean slides using DAPI Fluoromount G (Southern Biotech) or Prolong Gold.

Confocal imaging: Cells were imaged on a Nikon Ti-E microscope with a CSU-X1 Yokogawa spinning-disk confocal scan head (PerkinEImer, Wellesley, MA), an Andor iXon+ electron-multiplying charge-coupled device camera (Andor), using a 100X/1.4 NA objective lens. 
Z-step size was set at $0.2 \mu \mathrm{m}$.

557 Laser powers and exposures were chosen to ensure that the fluorescent signal would not be 558 saturated and were adjusted depending on the fluorescent signal. For imaging microtubules with 559 a Dylight 488 labeled secondary antibody, images were acquired using a $488 \mathrm{~nm}$ laser at $10.2 \%$ 560 power; for imaging DNA, the $405 \mathrm{~nm}$ laser was used at $40.2 \%$ power.

594 Line scan analysis (Fig. 4D, Fig. S6) was completed using confocal images of spindles that 595 were oriented parallel to the coverslip. Image stacks were first transformed into sum intensity 596 projections in Fiji. Then, the line width was matched to the width of the spindle, and a line (or 597 segmented line in the case of bent anaphase/telophase spindles) was drawn to include the 
entire spindle length, with a short length of background at each end. The "plot profile" tool in Fiji was then used to extract the average pixel intensity along the line for tubulin and DNA staining. These values were normalized to the average intensity of an area of the cell adjacent to the spindle, which was set to 1 . The spindle lengths were also normalized such that "0" represents the midpoint of the spindle. To determine the relative quantity of DNA and tubulin in these spindles (Fig. 4E), the area under the linescan-generated curves was calculated using GraphPad Prism 8 software, using a baseline level of 1.

Analysis of spindle twist: To characterize the shape of microtubule bundles, we manually tracked individual bundles of vertically oriented spindles, and horizontally oriented spindles whose image stacks were first transformed into vertical (end-on) orientation, using Multipoint tool in Fiji. As microtubule bundles appear as spots in a spindle cross-section, each point was placed at the center of the signal and its $x, y, z$ coordinates were saved. Moving up and down through the z-stack helped to determine this point. Each bundle was tracked through all z-planes where it was visible. Positions of the spindle poles were also determined, as the spots in the center of the end points of all bundles in the plane beyond the bundle ends. Coordinates of bundles and poles were transformed so that both poles are on the z-axis.

To describe the shape of a microtubule bundle, we fit a plane to the points representing the bundle. Subsequently, we fit a circle that lies in this plane to the same points. These fits were used to calculate the curvature and twist of the bundle as follows: (i) The curvature is calculated as one over the radius, and (ii) the twist is calculated as the angle between the plane and the z-axis divided by the mean distance of these points from the z-axis. Bundle length was calculated as the length of the projection of the bundle trace onto the pole-to-pole axis. For detailed descriptions of this method, see (Ivec et al., 2021).

Transmission Electron Microscopy: Cells were fixed overnight at $4{ }^{\circ} \mathrm{C}$ in $2.5 \%$ glutaraldehyde $+100 \mathrm{mM}$ sodium cacodylate, then rinsed and stored in $100 \mathrm{mM}$ sodium cacodylate overnight. Samples were then rinsed in $100 \mathrm{mM}$ sodium cacodylate buffer, $\mathrm{pH}$ 7.4, three times for 10 minutes per wash. Cells were post fixed in $1 \%$ aqueous osmium tetroxide (Electron Microscopy Sciences) in $100 \mathrm{mM}$ sodium cacodylate buffer overnight at $4{ }^{\circ} \mathrm{C}$. Cells were then rinsed twice in water for 10 min per wash, before en bloc staining with $1 \%$ uranyl acetate (Electron Microscopy Sciences) in water for 1 hour at room temperature. Cells were rinsed 3 times in water, for 10 min per wash. Cells were then subjected to a graded ethanol dehydration series as follows with 15 min washes at each of the following ethanol concentrations: $50 \%, 70 \%, 80 \%, 90 \%, 95 \%$, followed by two ten minute washes in $100 \%$ ethanol. Cells were quickly rinsed in propylene oxide, then infiltrated with $50 \%$ resin (Araldite 502/Embed-12, Electron Microscopy Sciences) and propylene oxide overnight. Cells were then incubated for 6-12 hours in each of the following resin concentrations: $70 \%, 85 \%, 95 \%$, and $100 \%$ followed by embedding in $100 \%$ resin at $60{ }^{\circ} \mathrm{C}$ for 4 days. $\sim 70 \mathrm{~nm}$ thin sections were cut using an RMC PowerTime XL Ultramicrotome with a Diatome diamond knife, and were transferred to copper grids. Sections were post stained with $1 \%$ uranyl acetate for $6 \mathrm{~min}$, and lead citrate for $2 \mathrm{~min}$. Images were taken using a JEOL JEM-200CX transmission electron microscope. 


\section{ACKNOWLEDGEMENTS}

We thank Nenad Pavin for discussions about the analysis of spindle twist, Shane Hussey for assistance with sequence analysis, Joshua Rafferty and Shadi Mahjoum for technical assistance, Laura Katz (Smith College) for assistance with the PhyloToL pipeline, and Sandra Balduaf (Uppsala University) for Acrasis tubulin sequences. We thank Chandler Fulton (Brandeis University) for strains and advice. We thank Alfredo Guzman for designing primers, Ravi Ranjan (University of Massachusetts, Genomics Resource Laboratory) for RNA extractions, and Madelaine Bartlett (UMass) and Courtney Babbitt (UMass) for qPCR equipment. We thank Andrew Kennard (UMass) and Tom Maresca (UMass) for comments on the manuscript. Light microscopy data was gathered in the Light Microscopy Facility and Nikon Center of Excellence at the Institute for Applied Life Sciences, UMass Amherst. We thank Kasia Hammar (Marine Biological Laboratory) for assistance with Transmission Electron Microscopy. 3D reconstructions were generated in UCSF ChimeraX, developed by the Resource for Biocomputing, Visualization, and Informatics at the University of California, San Francisco, with support from National Institutes of Health R01-GM129325 and the Office of Cyber Infrastructure and Computational Biology, National Institute of Allergy and Infectious Diseases. This work was supported by the National Institute Of Allergy And Infectious Diseases of the National Institutes of Health under Award Number F32AI150057 to K.B.V. and Award Number 1R21Al139363 to L.K.F.-L., and a Smith Family Foundation Award for Excellence in Biomedical Science to L.K.F.-L. The work of doctoral students M.T. and A.I. has been supported by the "Young researchers' career development project - training of doctoral students" of the Croatian Science Foundation. M.T., A.I. and I.M.T. acknowledge the support of the European Research Council (ERC Consolidator Grant, GA Number 647077) and the Croatian Science Foundation (HRZZ project IP2019-04-5967). Work in L.M.R.'s lab was supported by the NSF (MCB-1615938 and MCB-2017687) and the Robert A Welch Foundation (I-1908). 


\section{REFERENCES}

664 Akiyoshi, B. and Gull, K. (2014) 'Discovery of unconventional kinetochores in kinetoplastids', 665 Cell, 156(6), pp. 1247-1258.

666 Alushin, G. M. et al. (2014) 'High-resolution microtubule structures reveal the structural transitions in aß-tubulin upon GTP hydrolysis', Cell, 157(5), pp. 1117-1129.

Anisimova, M. et al. (2011) 'Survey of branch support methods demonstrates accuracy, power, and robustness of fast likelihood-based approximation schemes', Systematic biology, 60(5), pp. 685-699.

Brunet, S. et al. (1999) 'Kinetochore fibers are not involved in the formation of the first meiotic spindle in mouse oocytes, but control the exit from the first meiotic M phase', The Journal of cell biology, 146(1), pp. 1-12.

Cerón-Romero, M. A. et al. (2019) 'PhyloToL: A Taxon/Gene-Rich Phylogenomic Pipeline to Explore Genome Evolution of Diverse Eukaryotes', Molecular biology and evolution, 36(8), pp. 1831-1842.

Chung, S. et al. (2002) 'Cloning and characterization of a divergent alpha-tubulin that is expressed specifically in dividing amebae of Naegleria gruberi', Gene, 293(1-2), pp. 77-86.

Danlasky, B. M. et al. (2020) 'Evidence for anaphase pulling forces during C. elegans meiosis', The Journal of cell biology, 219(12). doi: 10.1083/jcb.202005179.

D’Archivio, S. and Wickstead, B. (2017) 'Trypanosome outer kinetochore proteins suggest conservation of chromosome segregation machinery across eukaryotes', The Journal of cell biology, 216(2), pp. 379-391.

Drinnenberg, I. A. and Akiyoshi, B. (2017) 'Evolutionary Lessons from Species with Unique Kinetochores', Progress in molecular and subcellular biology, 56, pp. 111-138.

Dumont, J., Oegema, K. and Desai, A. (2010) 'A kinetochore-independent mechanism drives anaphase chromosome separation during acentrosomal meiosis', Nature cell biology, 12(9), pp. 894-901.

Eshun-Wilson, L. et al. (2019) 'Effects of a-tubulin acetylation on microtubule structure and stability', Proceedings of the National Academy of Sciences of the United States of America, 116(21), pp. 10366-10371.

Fritz-Laylin, L. K., Assaf, Z. J., et al. (2010) 'Naegleria gruberi de novo basal body assembly occurs via stepwise incorporation of conserved proteins', Eukaryotic cell, 9(6), pp. 860-865.

Fritz-Laylin, L. K., Prochnik, S. E., et al. (2010) 'The genome of Naegleria gruberi illuminates early eukaryotic versatility', Cell, 140(5), pp. 631-642.

Fritz-Laylin, L. K. et al. (2011) 'The Naegleria genome: a free-living microbial eukaryote lends unique insights into core eukaryotic cell biology', Research in microbiology, 162(6), pp. 607-618. 
Fritz-Laylin, L. K. and Cande, W. Z. (2010) 'Ancestral centriole and flagella proteins identified by analysis of Naegleria differentiation', Journal of cell science, 123(Pt 23), pp. 4024-4031.

Fulton, C. (1983) 'Macromolecular syntheses during the quick-change act of Naegleria', The Journal of protozoology, 30(2), pp. 192-198.

Fulton, C. (1993) 'Naegleria: A Research Partner For Cell and Developmental Biology 1', The Journal of eukaryotic microbiology, 40(4), pp. 520-532.

Fulton, C. and Dingle, A. D. (1971) 'Basal bodies, but not centrioles, in Naegleria', The Journal of cell biology, 51(3), pp. 826-836.

Fulton, C. and Guerrini, A. M. (1969) 'Mitotic synchrony in Nalegleria amebae', Experimental cell research, 56(2), pp. 194-200.

Fulton, C. and Kowit, J. D. (1975) 'Programmed synthesis of flagellar tubulin during cell differentiation in Naegleria', Annals of the New York Academy of Sciences, 253, pp. 318-332.

Fulton, C. and Simpson, P. A. (1976) 'Selective synthesis and utilization of flagellar tubulin. The multi-tubulin hypothesis', Cell motility, 3, pp. 987-1005.

González-Robles, A. et al. (2009) 'Naegleria fowleri: light and electron microscopy study of mitosis', Experimental parasitology, 122(3), pp. 212-217.

Guindon, S. et al. (2010) 'New algorithms and methods to estimate maximum-likelihood phylogenies: assessing the performance of PhyML 3.0', Systematic biology, 59(3), pp. 307-321.

Gupta, M. L., Jr et al. (2003) 'Understanding tubulin-Taxol interactions: mutations that impart Taxol binding to yeast tubulin', Proceedings of the National Academy of Sciences of the United States of America, 100(11), pp. 6394-6397.

Heald, R. et al. (1996) 'Self-organization of microtubules into bipolar spindles around artificial chromosomes in Xenopus egg extracts', Nature, 382(6590), pp. 420-425.

Herman, E. K. et al. (2020) 'A comparative 'omics approach to candidate pathogenicity factor discovery in the brain-eating amoeba Naegleria fowleri', bioRxiv. bioRxiv. doi: 10.1101/2020.01.16.908186.

Heuser, M. and Razavi, L. (1970) 'Chapter 13 Amebo-flagellates as Research Partners: The Laboratory Biology of Naegleria and Tetramitus', in Prescott, D. M. (ed.) Methods in Cell Biology. Academic Press, pp. 341-476.

Hoang, D. T. et al. (2018) 'UFBoot2: Improving the Ultrafast Bootstrap Approximation', Molecular biology and evolution, 35(2), pp. 518-522.

van Hooff, J. J. et al. (2017) 'Evolutionary dynamics of the kinetochore network in eukaryotes as revealed by comparative genomics', EMBO reports, 18(9), pp. 1559-1571.

Hoyle, H. D. and Raff, E. C. (1990) 'Two Drosophila beta tubulin isoforms are not functionally equivalent', The Journal of cell biology, 111(3), pp. 1009-1026. 
Inoué, S. and Salmon, E. D. (1995) 'Force generation by microtubule assembly/disassembly in mitosis and related movements', Molecular biology of the cell, 6(12), pp. 1619-1640.

Ivec, A. et al. (2021) 'Measurement of curvature and twist of microtubule bundles in the mitotic spindle', bioRxiv. doi: 10.1101/2021.01.02.425081.

Johnson, K. A. (1998) 'The axonemal microtubules of the Chlamydomonas flagellum differ in tubulin isoform content', Journal of cell science, 111 ( Pt 3), pp. 313-320.

Kajtez, J. et al. (2016) 'Overlap microtubules link sister k-fibres and balance the forces on bi-oriented kinetochores', Nature communications, 7, p. 10298.

Kalyaanamoorthy, S. et al. (2017) 'ModelFinder: fast model selection for accurate phylogenetic estimates', Nature methods, 14(6), pp. 587-589.

Kapoor, T. M. et al. (2006) 'Chromosomes can congress to the metaphase plate before biorientation', Science, 311(5759), pp. 388-391.

Kowit, J. D. and Fulton, C. (1974a) 'Programmed synthesis of tubulin for the flagella that develop during cell differentiation in Naegleria gruberi', Proceedings of the National Academy of Sciences of the United States of America, 71(7), pp. 2877-2881.

Kowit, J. D. and Fulton, C. (1974b) 'Purification and properties of flagellar outer doublet tubulin from Naegleria gruberi and a radioimmune assay for tubulin', The Journal of biological chemistry, 249(11), pp. 3638-3646.

Lai, E. Y. et al. (1979) 'Programmed appearance of translatable flagellar tubulin mRNA during cell differentiation in Naegleria', Cell, 17(4), pp. 867-878.

Lai, E. Y., Remillard, S. P. and Fulton, C. (1988) 'The alpha-tubulin gene family expressed during cell differentiation in Naegleria gruberi', The Journal of cell biology, 106(6), pp. 2035-2046.

Lee, J. H. and Walsh, C. J. (1988) 'Transcriptional regulation of coordinate changes in flagellar mRNAs during differentiation of Naegleria gruberi amebae into flagellates', Molecular and cellular biology, 8(6), pp. 2280-2287.

Letunic, I. and Bork, P. (2019) 'Interactive Tree Of Life (iTOL) v4: recent updates and new developments', Nucleic acids research. doi: 10.1093/nar/gkz239.

Livak, K. J. and Schmittgen, T. D. (2001) 'Analysis of relative gene expression data using real-time quantitative PCR and the 2(-Delta Delta C(T)) Method', Methods , 25(4), pp. 402-408.

Madeira, F. et al. (2019) 'The EMBL-EBI search and sequence analysis tools APIs in 2019', Nucleic acids research, 47(W1), pp. W636-W641.

Manenica, M. et al. (2020) 'Augmin regulates kinetochore tension and spatial arrangement of spindle microtubules by nucleating bridging fibers', bioRxiv. doi: 10.1101/2020.09.10.291740.

8 Mastronarde, D. N. et al. (1993) 'Interpolar spindle microtubules in PTK cells', The Journal of 
cell biology, 123(6 Pt 1), pp. 1475-1489.

792 Raff, E. C. 793 1-10.

794 Savage, C. et al. (1989) 'mec-7 is a beta-tubulin gene required for the production of

795 15-protofilament microtubules in Caenorhabditis elegans', Genes \& development, 3(6), pp.

796 870-881.

797

Matthews, K. A., Rees, D. and Kaufman, T. C. (1993) 'A functionally specialized alpha-tubulin is required for oocyte meiosis and cleavage mitoses in Drosophila', Development, 117(3), pp. 977-991.

Mclntosh, J. R., Molodtsov, M. I. and Ataullakhanov, F. I. (2012) 'Biophysics of mitosis', Quarterly reviews of biophysics, 45(2), pp. 147-207.

Meza, I., Talamás-Rohana, P. and Vargas, M. A. (2006) 'The cytoskeleton of Entamoeba histolytica: structure, function, and regulation by signaling pathways', Archives of medical research, 37(2), pp. 234-243.

Minh, B. Q. et al. (2020) 'IQ-TREE 2: New Models and Efficient Methods for Phylogenetic Inference in the Genomic Era', Molecular biology and evolution, 37(5), pp. 1530-1534.

Mirarab, S. et al. (2015) 'PASTA: Ultra-Large Multiple Sequence Alignment for Nucleotide and Amino-Acid Sequences', Journal of computational biology: a journal of computational molecular cell biology, 22(5), pp. 377-386.

Mullen, T. J., Davis-Roca, A. C. and Wignall, S. M. (2019) 'Spindle assembly and chromosome dynamics during oocyte meiosis', Current opinion in cell biology, 60, pp. 53-59.

Novak, M. et al. (2018) 'The mitotic spindle is chiral due to torques within microtubule bundles', Nature communications, 9(1), p. 3571.

Pei, J. and Grishin, N. V. (2001) 'AL2CO: calculation of positional conservation in a protein sequence alignment', Bioinformatics , 17(8), pp. 700-712.

Pettersen, E. F. et al. (2021) 'UCSF ChimeraX: Structure visualization for researchers, educators, and developers', Protein science: a publication of the Protein Society, 30(1), pp. $70-82$.

Schindelin, J. et al. (2012) 'Fiji: an open-source platform for biological-image analysis', Nature methods, 9(7), pp. 676-682.

Siddiqui, R. et al. (2016) 'Biology and pathogenesis of Naegleria fowleri', Acta tropica, 164, pp. 375-394.

Simunić, J. and Tolić, I. M. (2016) 'Mitotic Spindle Assembly: Building the Bridge between Sister K-Fibers', Trends in biochemical sciences, 41(10), pp. 824-833.

3 Tolić, I. M., Novak, M. and Pavin, N. (2019) 'Helical Twist and Rotational Forces in the Mitotic 
804 Spindle', Biomolecules, 9(4). doi: 10.3390/biom9040132.

805 Velle, K. B. and Fritz-Laylin, L. K. (2020) 'Conserved actin machinery drives

806 microtubule-independent motility and phagocytosis in Naegleria', The Journal of cell biology,

807 219(11). doi: 10.1083/jcb.202007158.

808 Vemu, A. et al. (2017) 'Tubulin isoform composition tunes microtubule dynamics', Molecular 809 biology of the cell, 28(25), pp. 3564-3572.

810 Vukušić, K. et al. (2017) 'Microtubule Sliding within the Bridging Fiber Pushes Kinetochore

811 Fibers Apart to Segregate Chromosomes', Developmental cell, 43(1), pp. 11-23.e6.

812 Vukušić, K., Buđa, R. and Tolić, I. M. (2019) 'Force-generating mechanisms of anaphase in 813 human cells', Journal of cell science, 132(18). doi: 10.1242/jcs.231985.

814 Walsh, C. J. (2007) 'The role of actin, actomyosin and microtubules in defining cell shape during 815 the differentiation of Naegleria amebae into flagellates', European journal of cell biology, 86(2),

816 pp. 85-98.

817 Walsh, C. J. (2012) 'The structure of the mitotic spindle and nucleolus during mitosis in the 818 amebo-flagellate Naegleria', PloS one, 7(4), p. e34763.

819 Wilson, P. G. and Borisy, G. G. (1997) 'Evolution of the multi-tubulin hypothesis', BioEssays:

820 news and reviews in molecular, cellular and developmental biology, 19(6), pp. 451-454. 
bioRxiv preprint doi: https://doi.org/10.1101/2021.02.23.432318; this version posted February 23, 2021. The copyright holder for this preprint (which was not certified by peer review) is the author/funder, who has granted bioRxiv a license to display the preprint in perpetuity. It is made

A

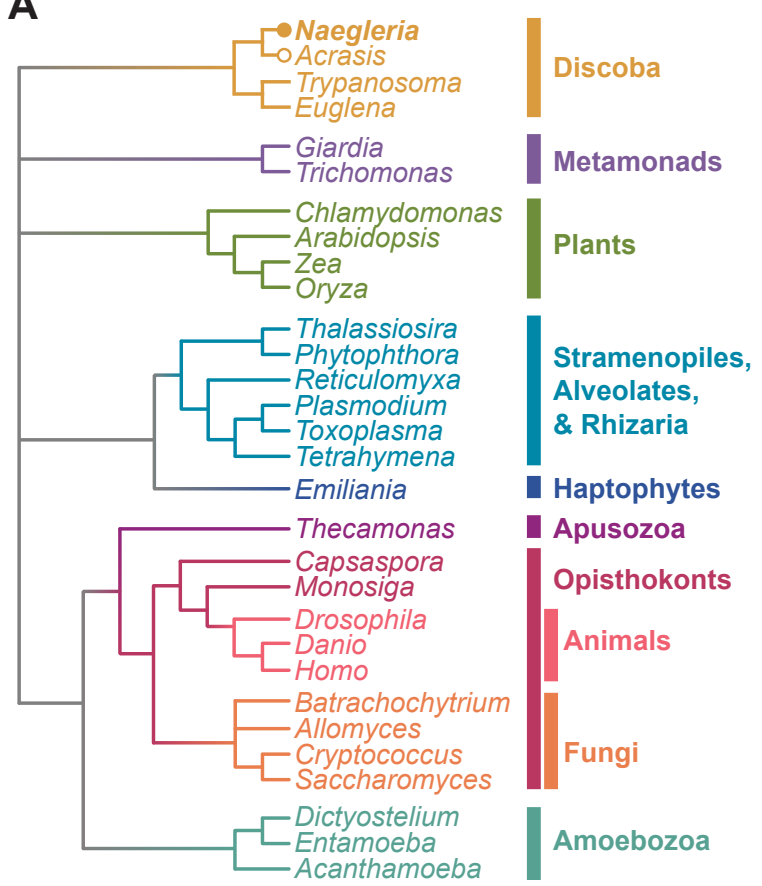

B

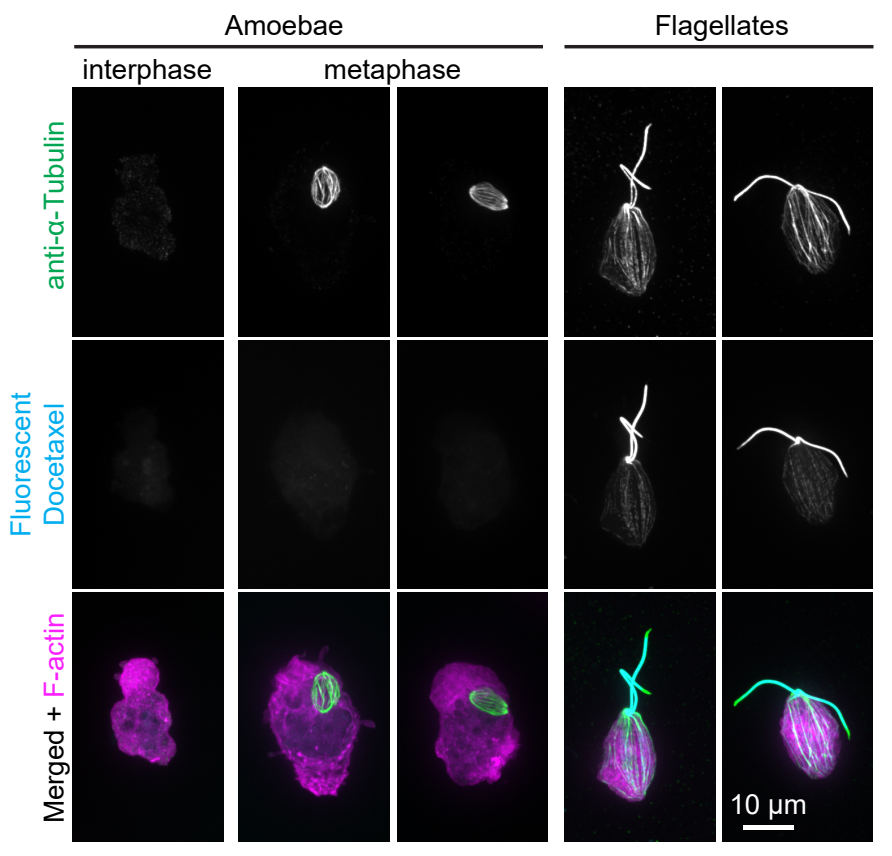

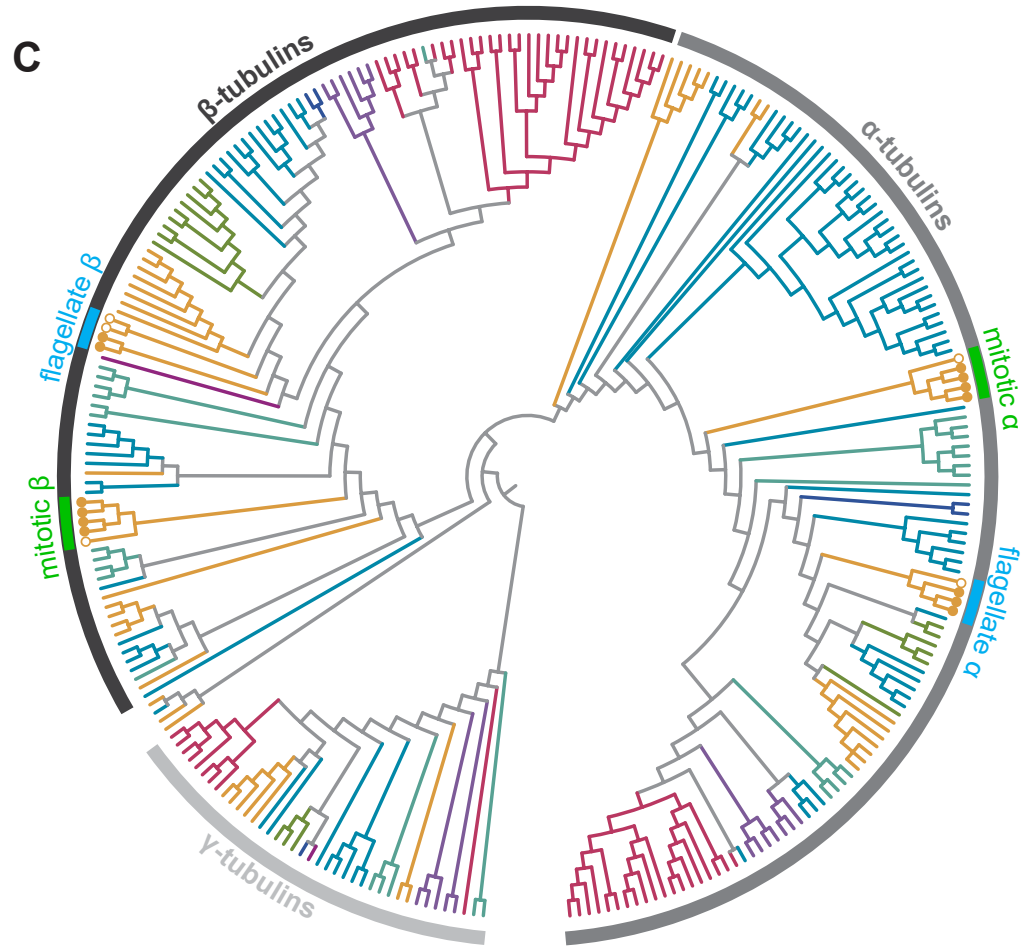

D
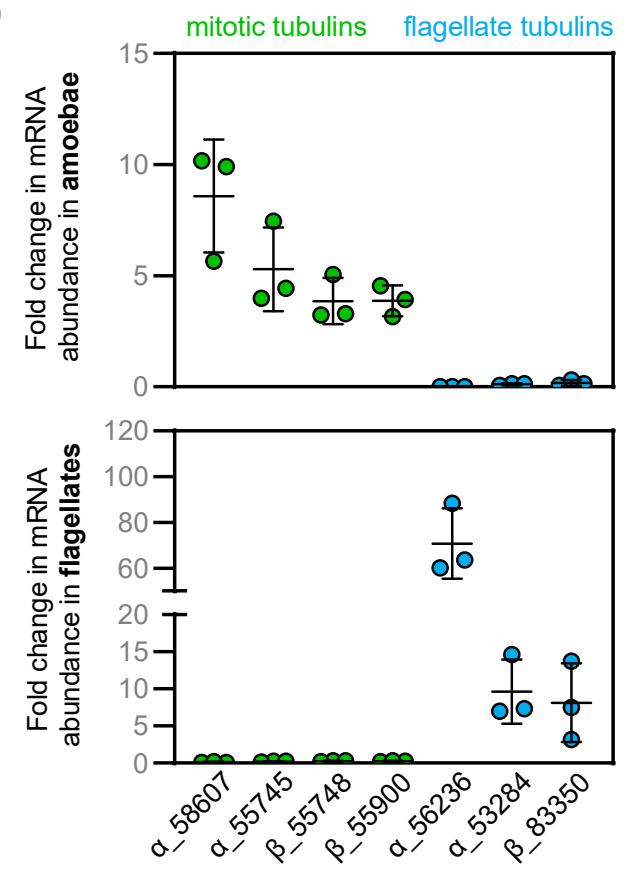

Figure 1. Naegleria has flagellate and mitotic microtubule arrays composed of distinct tubulins. (A) The evolutionary relationships between Naegleria and other eukaryotes are shown using a cladogram (branch lengths are meaningless) modified from Velle and Fritz-Laylin, 2019. (B) Amoebae from a growing population (left), or flagellates from a differentiated population (right), were fixed and stained with antibodies (anti-alpha tubulin clone DM1A, green) and Tubulin Tracker (Fluorescent Docetaxel, cyan) to detect microtubules, and Alexa Fluor 488 conjugated Phalloidin to label F-actin (magenta). Maximum intensity projections of cells are shown. (C) The evolutionary relationship of gamma, alpha, and beta tubulins from the species in panel A are shown using a cladogram (using the color scheme from A, see Fig. S1 for the full tree). The tree is rooted on gamma tubulins, and shows mitotic (green) and flagellate (blue) tubulins from Naegleria (closed circles) and Acrasis (open circles). (D) The fold changes in tubulin mRNA in amoebae compared to flagellates (top) or flagellates compared to amoebae (bottom) were calculated from data reported in Fritz-Laylin and Cande, 2010. Each point represents one experimental replicate, and lines denote the average +/- standard deviation (SD). Tubulins are labeled with JGI identification numbers. 
A
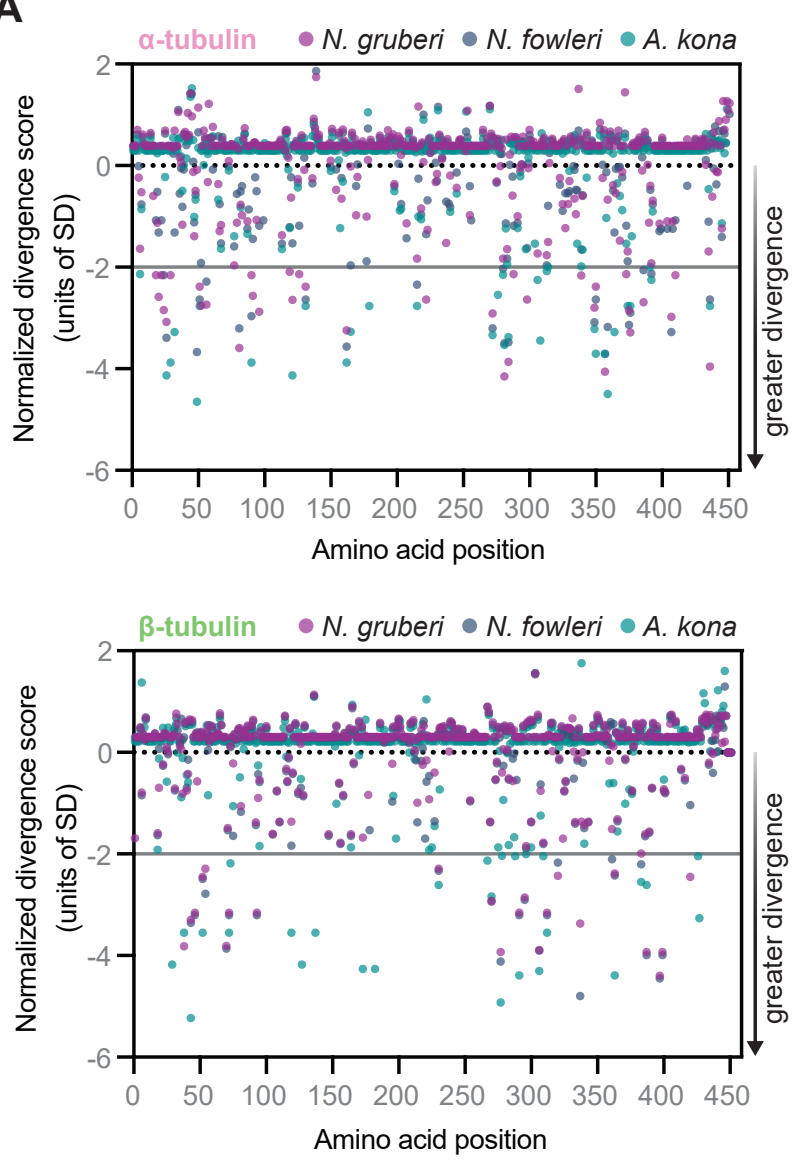

B

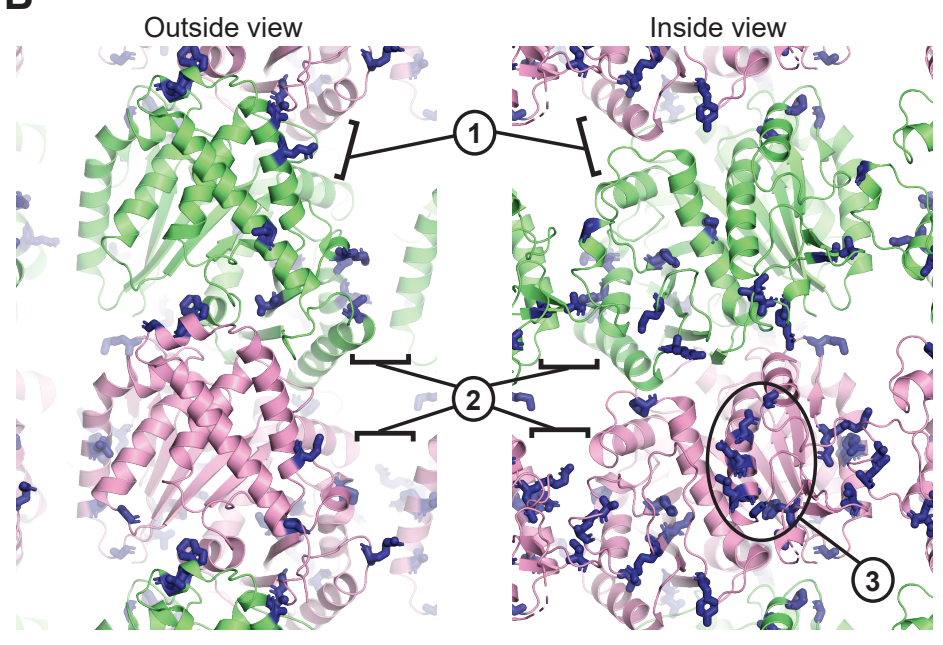

C

(1)

(2)

\begin{tabular}{cccc} 
& $\begin{array}{c}\text { \% Divergent } \\
\text { (overall) }\end{array}$ & $\begin{array}{c}\text { \% Divergent } \\
\text { (Longitudinal) }\end{array}$ & $\begin{array}{c}\text { \% Divergent } \\
\text { (Lateral) }\end{array}$ \\
\hline N. gruberi & $\alpha 8.2$ & $\alpha 5.7$ & $\alpha 12.9$ \\
& $\beta 5.5$ & $\beta 5.8$ & $\beta 10.5$ \\
\hline N. fowleri & $\alpha 5.4$ & $\alpha 5.7$ & $\alpha 9.7$ \\
& $\beta 4.1$ & $\beta 5.8$ & $\beta 7.0$ \\
\hline A. kona & $\alpha 7.0$ & $\alpha 8.0$ & $\alpha 16.1$ \\
& $\beta 5.5$ & $\beta 8.1$ & $\beta 8.8$
\end{tabular}

Figure 2. Comparative analysis of evolutionary divergence for mitotic and flagellate tubulins. (A) Plots of the normalized divergence score (see Methods) as a function of amino acid position for $\alpha$-tubulin (top) and $\beta$-tubulin (bottom). Lower scores indicate positions where mitotic tubulins show increased divergence relative to flagellate tubulins. The analysis was performed on three species: $N$. gruberi (lavender), $N$. fowleri (navy), and $A$. kona (teal). The horizontal gray line indicates the two standard deviation cutoff we used to identify especially divergent sites. (B) Structural context of the sites with increased divergence in the mitotic tubulins. Side-chain positions for the $N$. gruberi amino acids identified in (A) are represented as sticks (blue) on a model of a $\beta$-tubulin in the microtubule lattice ( $\alpha$-tubulin: pink, $\beta$-tubulin: lime). 'Outside' and 'Inside' views of the lattice are shown, and longitudinal (labeled 1 ) and lateral (labeled 2) microtubule lattice contacts are indicated, as is the luminal (internal) surface of $\alpha$-tubulin (labeled 3). (C) Table summarizing the proportion of positions with elevated divergence near microtubule lattice interfaces. For all three species, there are more divergent positions in $\alpha$-tubulin compared to $\beta$-tubulin, and the divergence seems to be particularly enriched at the lateral interfaces. See Fig. S4 for details. 
bioRxiv preprint doi: https://doi.org/10.1101/2021.02.23.432318; this version posted February 23, 2021. The copyright holder for this preprint (which was not certified by peer review) is the author/funder, who has granted bioRxiv a license to display the preprint in perpetuity. It is made

A

A Prophase
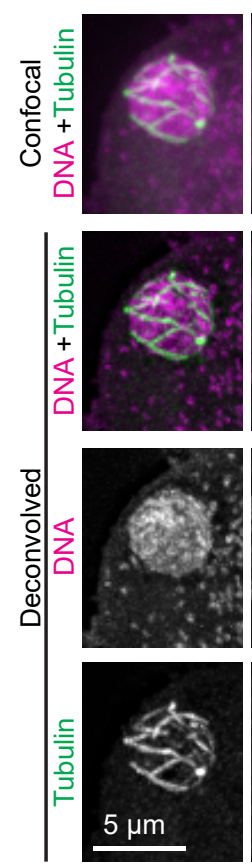

B

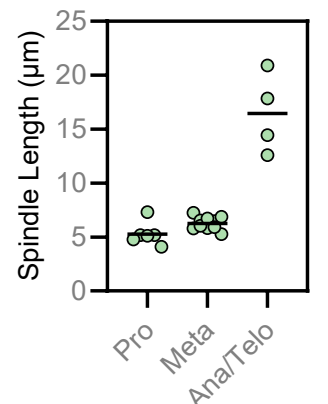

E
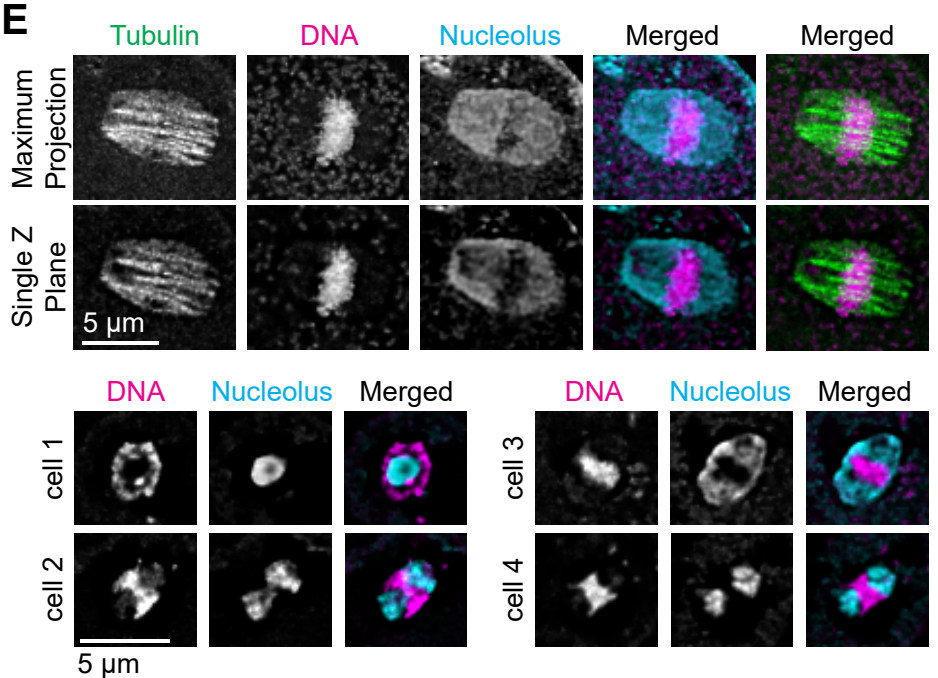

Metaphase

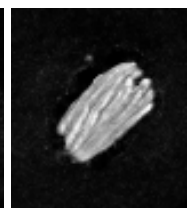

C
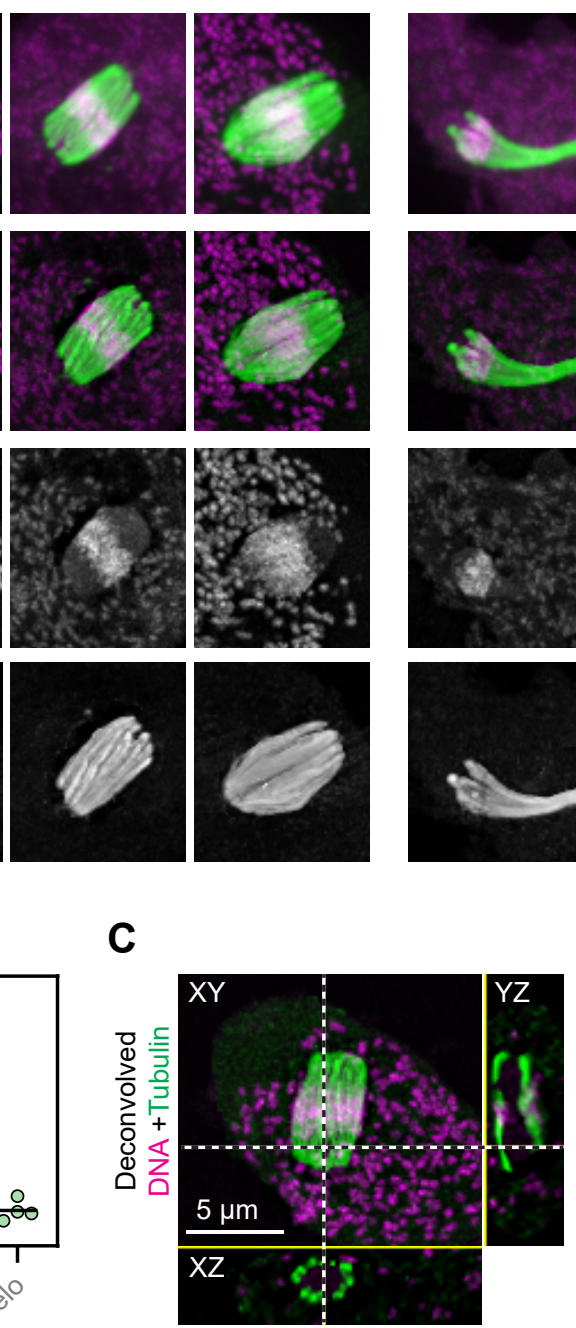

D

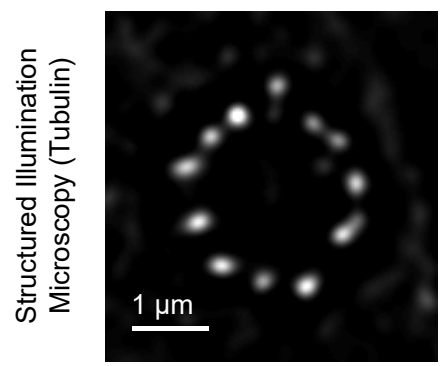

$\mathbf{F}$
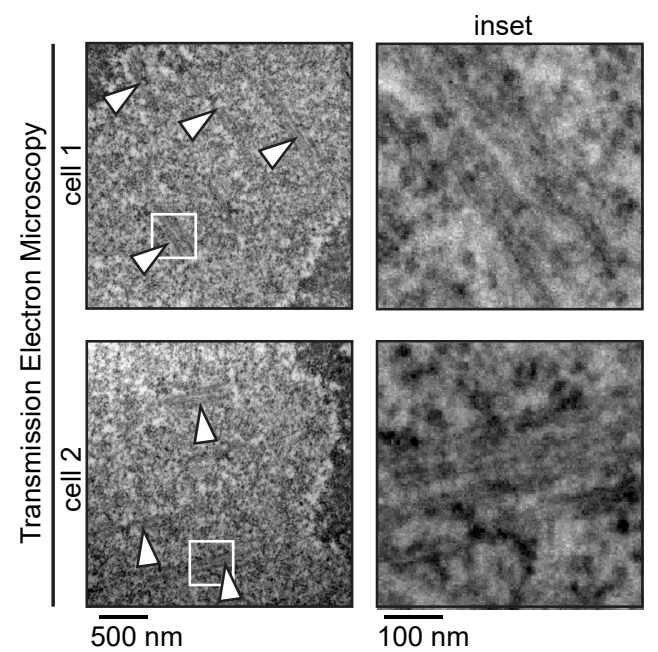

Figure 3. Naegleria's spindle is a barrel shape composed of bundles of microtubules that elongate as mitosis proceeds. (A) Asynchronously growing Naegleria amoebae were fixed and stained with anti-alpha tubulin clone DM1A (green) to detect microtubules, and DAPI to label DNA (magenta). Mitotic spindles were imaged using confocal microscopy (top row), and images were deconvolved using Autoquant software (bottom rows). Cells were classified as prophase, metaphase, or anaphase/telophase. (B) Quantification of maximum spindle length (left) and the spindle width at half the length (right). Each point represents one mitotic spindle, and lines indicate the averages (prophase, $n=6$; metaphase, $n=10$; anaphase/telophase, $n=4$ ). Spindles imaged and deconvolved as in (A). (C) 
Orthogonal views of a metaphase spindle (imaged and deconvolved as in A) lying in the plane of the coverslip; $X Z$ and $Y Z$ views generated in Fiji. (D) Structured illumination microscopy of a spindle lying perpendicular to the coverslip. (E) Confocal microscopy and deconvolution of nucleoli in mitotic Naegleria. Cells were fixed and stained to detect tubulin (YOL 1/34 antibody, green, top panels only), DNA (DAPI, magenta), and nucleolar protein (DE6 antibody, cyan). One maximum intensity projection is shown (top cell), while remaining images are single $Z$ planes. (F) Transmission electron microscopy of microtubule bundles in Naegleria; arrowheads indicate microtubule bundles and boxed regions (left) are shown as enlarged insets (right). 
bioRxiv preprint doi: https://doi.org/10.1101/2021.02.23.432318; this version posted February 23, 2021. The copyright holder for this preprint (which was not certified by peer review) is the author/funder, who has granted bioRxiv a license to display the preprint in perpetuity. It is made available under aCC-BY-NC 4.0 International license.

A

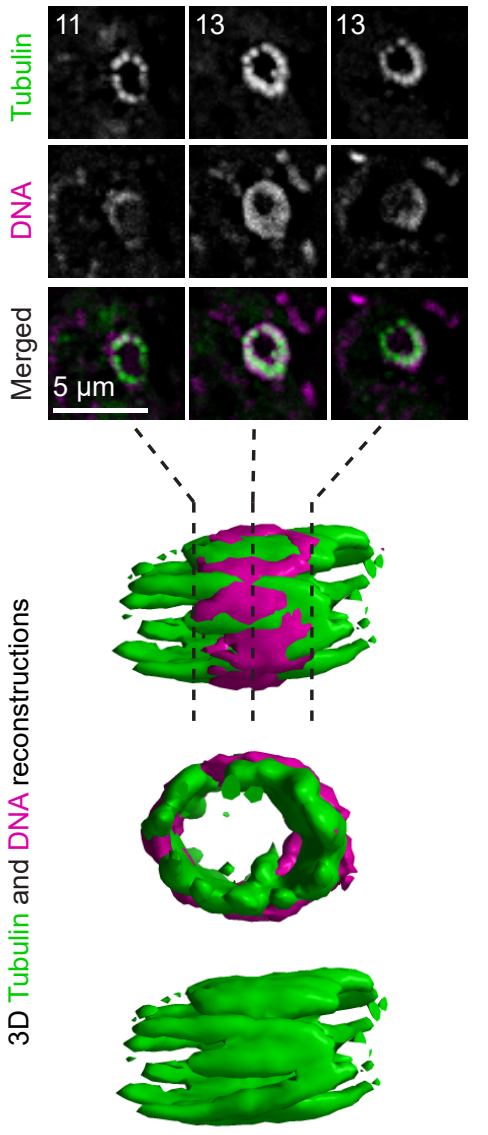

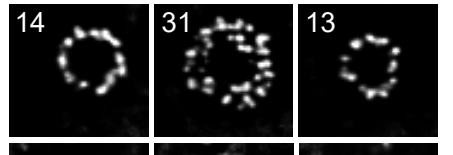
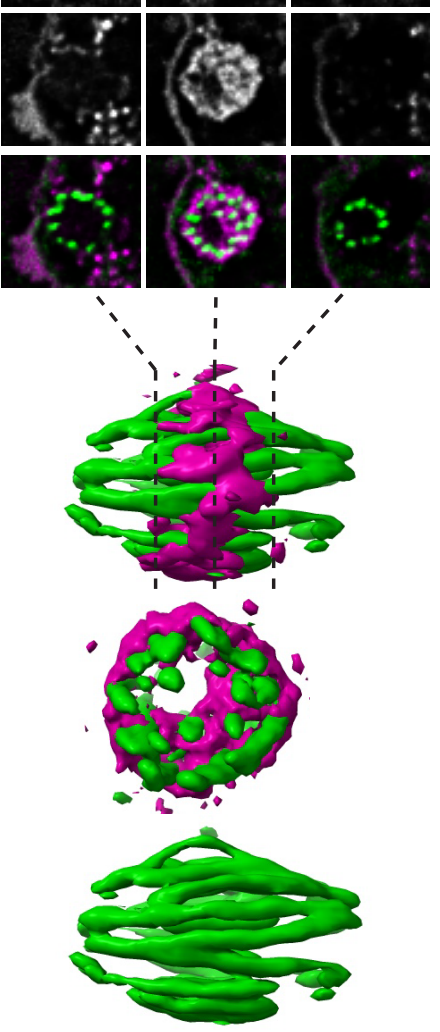
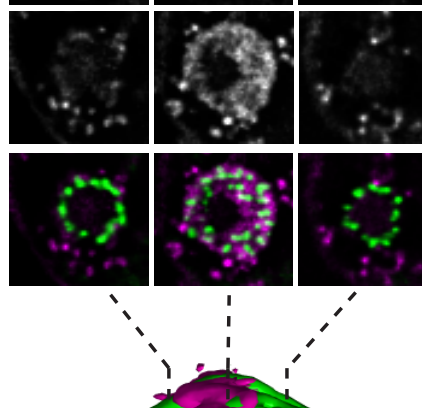

B
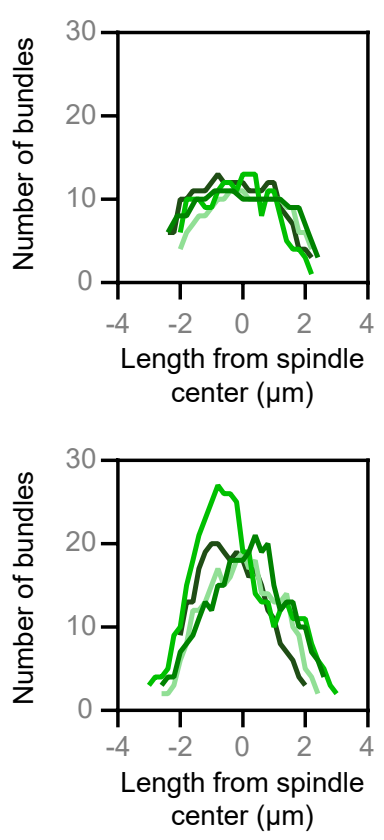

C

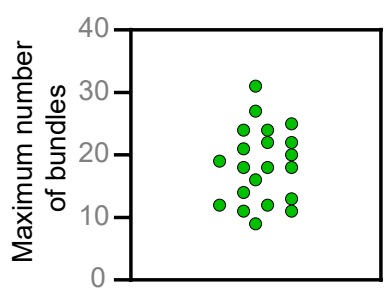

Metaphase
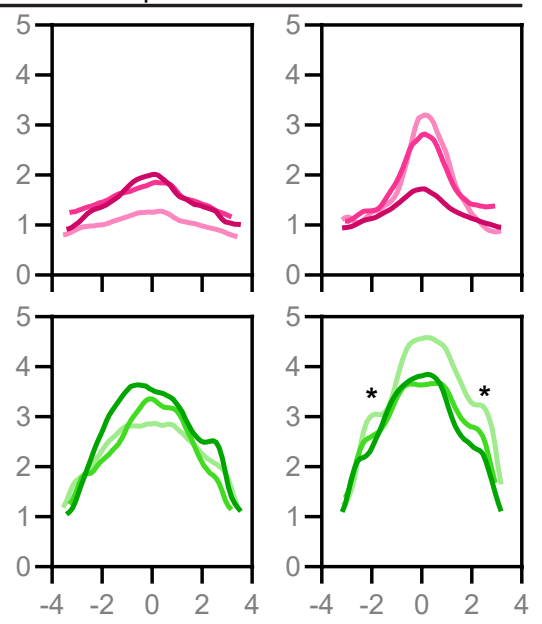

Anaphase/Telophase
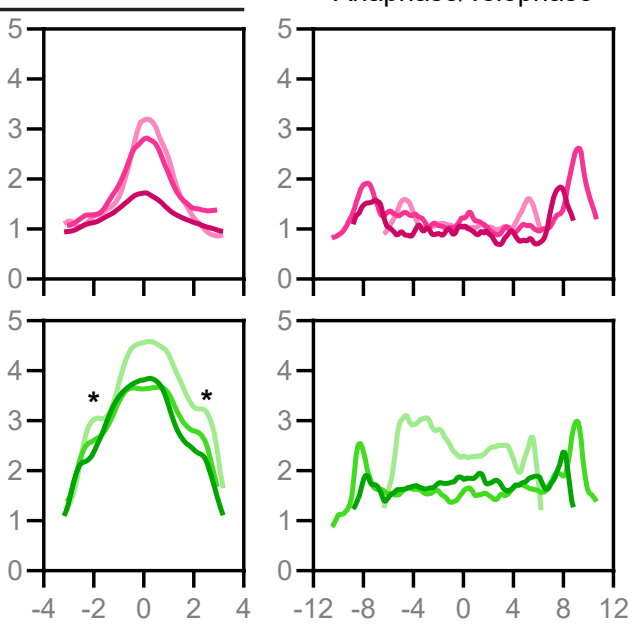

E

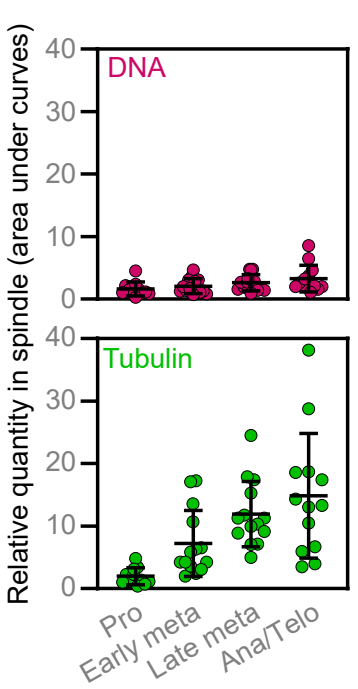

Figure 4. The number of microtubule bundles changes as mitosis proceeds. (A) Cells were fixed and stained with antibodies (anti-alpha tubulin clone DM1A, green) to detect microtubules, and DAPI to label DNA (magenta). Cells with spindles perpendicular to the coverslip were imaged using confocal microscopy and deconvolved using Autoquant software (top panels), and 3D reconstructions were rendered using ChimeraX software (bottom panels, not to scale). Individual Z planes are shown for slices approximately 25,50 , and $75 \%$ through the spindle for three representative cells. Numbers (upper left) indicate the number of distinct microtubule bundles in that position of the spindle. (B) The number of microtubule bundles throughout the spindle length in metaphase spindles, imaged as in (A). Some spindles (top) had a fairly consistent number of microtubule bundles throughout the spindle $(n=4)$, while other spindles (bottom) had a peak in the number of bundles towards the midpoint $(n=4)$. (C) The maximum number 
of microtubule bundles from confocal images of metaphase cells. (D) Line scans show the relative DNA and tubulin fluorescence intensity from sum intensity projections of spindles lying in the plane of the coverslip, imaged as in (A). Metaphase spindles were grouped based on the shapes of tubulin curves (no shoulders, left; unclear shoulders, center; two clear shoulders denoted by asterisks, right); three individual examples are shown in each panel (also see Fig. S6). (E) Quantification of DNA (top) or tubulin (bottom) from line scans obtained as in (D). Metaphase was categorized as early or late based on the presence (late) or absence (early) of shoulders (stages where no clear classification could be assigned were excluded). Each point represents the area under the curve for one spindle line scan, and lines indicate the mean $+/-$ SD. 
A
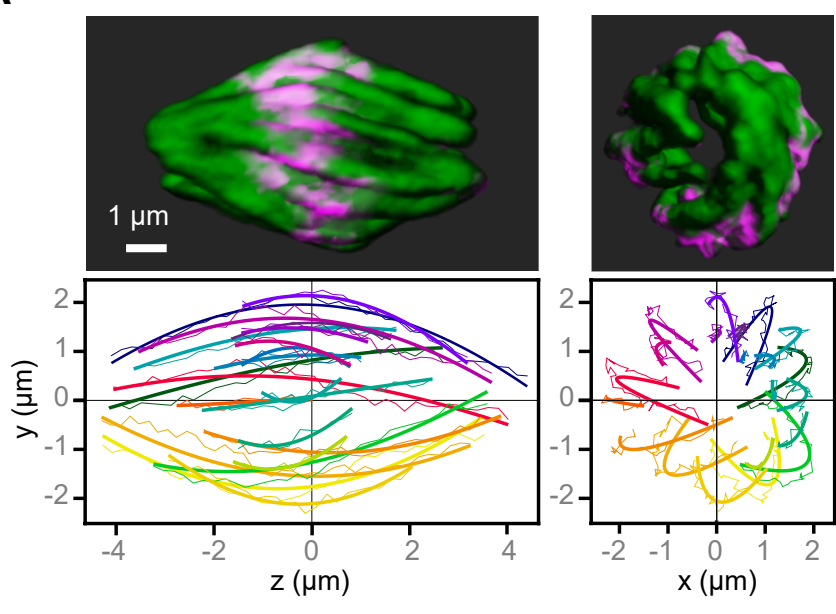

B
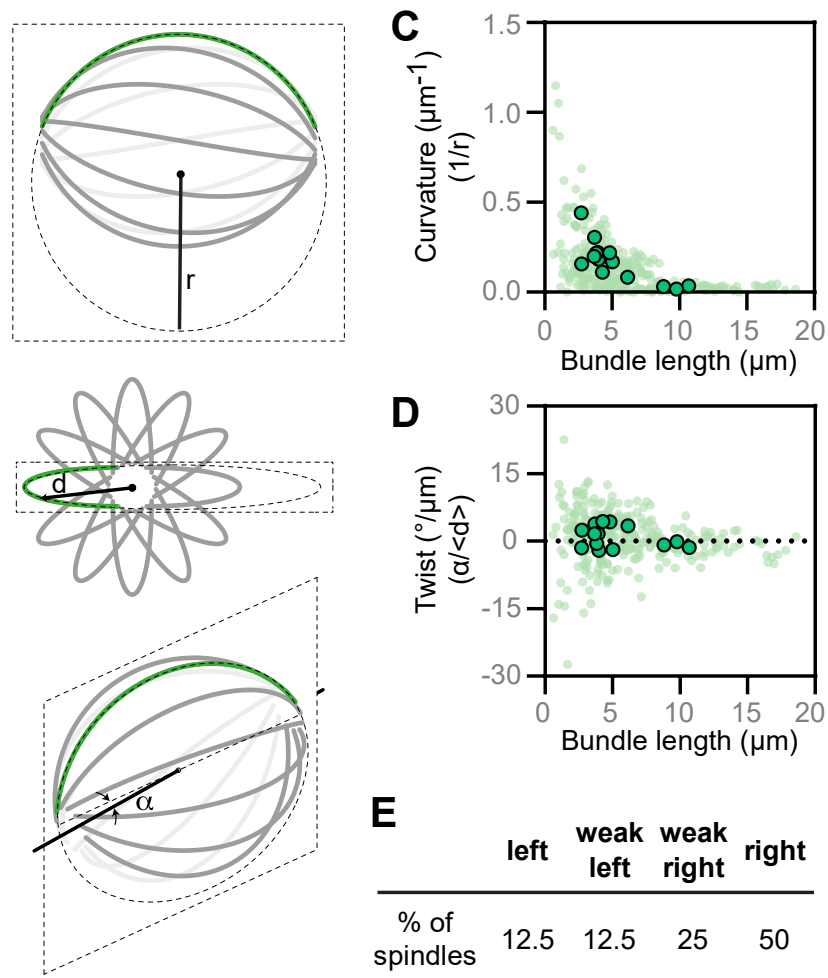

E

\begin{tabular}{|c|c|c|c|c|}
\hline 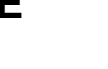 & left & $\begin{array}{c}\text { weak } \\
\text { left }\end{array}$ & $\begin{array}{l}\text { weak } \\
\text { right }\end{array}$ & right \\
\hline $\begin{array}{l}\% \text { of } \\
\text { pindles }\end{array}$ & 12.5 & 12.5 & 25 & 50 \\
\hline
\end{tabular}

Figure 5. Naegleria mitotic spindles are twisted. (A) A 3D reconstructed spindle (the same spindle shown in Fig. $4 \mathrm{~A}$, right) is shown from side and end-on view viewpoints. Microtubules are shown in green, and DNA is in magenta. Microtubule bundles were quantified from the side view (left graph) and end-on view (right graph). Each bundle is represented by a different color, thin lines mark the manually traced points along the bundle, and thick lines show circular arcs of the fitted circles. (B) A simplified scheme of a spindle is shown from the side (top), end-on (middle), and from an arbitrary angle (bottom). A microtubule bundle (green line) is fitted by a circle (dashed ellipse) of radius $(r)$. The angle ( $\alpha$ ) between the central spindle axis (solid line) and the plane in which the fitted circle lies (dashed parallelogram) is denoted. The distance (d) of the bundle from the central spindle axis is denoted. (C) The curvature of microtubule bundles is shown as a function of bundle length (measured along its pole to pole axis). Each small dot represents a single bundle within a spindle, while each larger dot represents the average for a spindle. (D) The twist of microtubule bundles is shown as a function of bundle length. Each small dot represents a single bundle within a spindle, while each larger dot represents the average for a spindle. (E) The percentage of spindles with right, weak right, left, or weak left handedness are shown (see Fig S7 for a breakdown of this analysis). 
bioRxiv preprint doi: https://doi.org/10.1101/2021.02.23.432318; this version posted February 23, 2021. The copyright holder for this preprint (which was not certified by peer review) is the author/funder, who has granted bioRxiv a license to display the preprint in perpetuity. It is made available under aCC-BY-NC 4.0 International license.

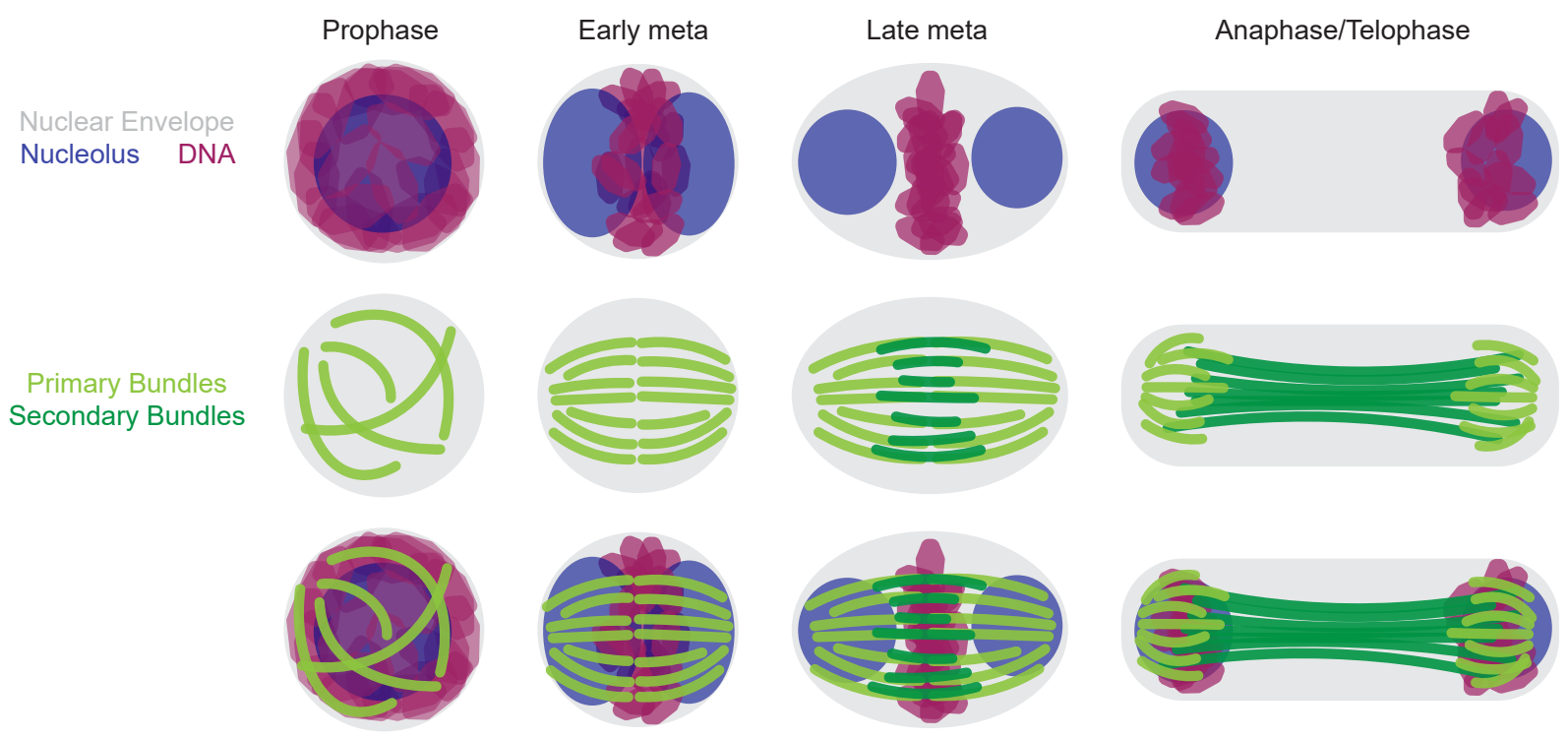

Figure 6. Model for mitosis in Naegleria. During prophase in Naegleria, bundles of microtubules form around a hollow sphere of DNA (magenta) which surrounds the single, round nucleolus (blue). In early metaphase, the DNA condenses into a disk, the nucleolus begins to divide and the microtubule bundles (light green) organize into a hollow, twisted barrel shape. In late metaphase, the DNA is further condensed, and the nucleolus resolves into two distinct spheres. A secondary set of microtubules forms in the equatorial region (dark green) adjacent to the primary bundles. During anaphase/telophase, the DNA is segregated to the two ends of the spindle and the spindle elongates. See text for details. 